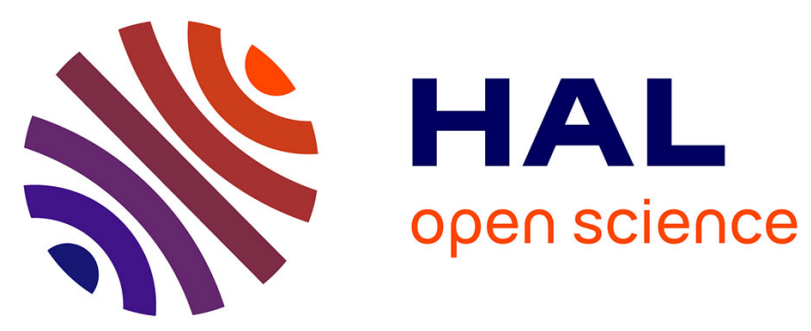

\title{
La perception des usagers de la voie publique du statut et des règles de circulation applicables aux aides à la mobilité motorisées
}

Jean-françois Bruneau, Pierre Maurice, Maud Quinones

\section{To cite this version:}

Jean-françois Bruneau, Pierre Maurice, Maud Quinones. La perception des usagers de la voie publique du statut et des règles de circulation applicables aux aides à la mobilité motorisées. RTS - Recherche Transports Sécurité, 2014, 2013 (04), pp.299-316. 10.4074/S0761898013400055 . hal-01670628

\section{HAL Id: hal-01670628 \\ https://hal.science/hal-01670628}

Submitted on 21 Dec 2017

HAL is a multi-disciplinary open access archive for the deposit and dissemination of scientific research documents, whether they are published or not. The documents may come from teaching and research institutions in France or abroad, or from public or private research centers.
L'archive ouverte pluridisciplinaire HAL, est destinée au dépôt et à la diffusion de documents scientifiques de niveau recherche, publiés ou non, émanant des établissements d'enseignement et de recherche français ou étrangers, des laboratoires publics ou privés. 


\title{
La perception des usagers de la voie publique du statut et des règles de circulation applicables aux aides à la mobilité motorisées
}

\author{
The perception of pedestrians, cyclists and vehicle drivers on the legal status and the roadway \\ rules related to personal mobility devices
}

\author{
Jean-François Bruneau ·Pierre Maurice · Maud Quinones
}

Reçu le 29 novembre 2012 ; accepté le 3 octobre 2013

(C) IFSTTAR et Éditions NecPlus 2013

\begin{abstract}
Résumé Considérés comme piétons en Amérique du Nord, et aussi comme cyclistes en Europe lorsqu'ils empruntent des voies autres que piétonnières, les utilisateurs d'Aides à la mobilité motorisées (AMM) ne font l'objet d'aucune règle au Code de la sécurité routière du Québec. Devant ce vide juridique, doublé d'un manque de connaissances sur les usagers, leurs besoins et leurs habitudes, l'Institut national de santé publique du Québec (INSPQ) a été mandaté par le Gouvernement pour produire un avis de santé publique sur cette question. Dans son avis, considérant la sécurité de tous les usagers de la route et le besoin d'autonomie des personnes ayant des incapacités à la marche, l'INSPQ recommande que les AMM soient autorisées sur les voies piétonnières, cyclables et routières. Pour valider ce cadre légal, un projet pilote (2011-2015), impliquant les instances municipales et gouvernementales, les forces policières et le milieu associatif, a été mis sur pied. Pour caractériser l'usage et pour évaluer les impacts sur la sécurité routière d'éventuelles règles de circulation devant être introduites, les utilisateurs d'AMM $(\mathrm{n}=186)$, les piétons $(\mathrm{n}=167)$, les cyclistes $(n=148)$ et les automobilistes $(n=109)$ ont été questionnés dans la rue ou au moyen d'un questionnaire auto-administré. Cet article décrit les résultats obtenus au regard des habitudes de déplacement des usagers d'AMM
\end{abstract}

\footnotetext{
Jean-François Bruneau $(\bowtie)$

Institut national de santé publique du Québec

2500, boul. de l'Université

Sherbrooke (Québec) Canada J1K 2R1

e-mail : jean-francois.bruneau@usherbrooke.ca

Pierre Maurice

Institut national de santé publique du Québec

2400, Avenue d'Estimauville

Québec (Québec) Canada G1E 7G9

e-mail : pierre.maurice@inspq.qc.ca

Maud Quinones

Institut national de santé publique du Québec

2400, Avenue d'Estimauville

Québec (Québec) Canada G1E 7G9
}

Université de Sherbrooke, Département de géomatique appliquée et de la perception de l'ensemble des usagers de la route concernant la circulation des AMM sur la voie publique (piétons, cyclistes et automobilistes). Les résultats illustrent le décalage parfois important entre les besoins de mobilité des utilisateurs d'AMM et l'opinion publique, et met en perspective certaines règles de circulation associées à une cohabitation plus difficile, telle l'utilisation de la chaussée.

Mots clés aide à la mobilité motorisée · piéton · cycliste . automobiliste $\cdot$ règle de circulation

Abstract Considered as pedestrians in North America, as well as cyclists in Europe, when riding on other infrastructures than sidewalks, users of Personal Mobility Devices (PMDs) are absent from the Quebec Roadway Safety Code. This lack of provincial regulation, along with the limited knowledge on safety issues, led the Quebec Government to ask the Institut national de santé publique du Québec (INSPQ) for a complete safety study. After a series of consultations, INSPQ recommended, for safety and public health issues, that PMDs should be allowed to circulate on sidewalks, roadways and cycling infrastructures. To evaluate this legal frame, a pilot-project (2011-2015) is ongoing with municipal and government officials, police and user organizations. To evaluate the safety implications of traffic laws that will eventually be introduced in the Code, a randomized sample of PMDs users $(\mathrm{n}=185)$, pedestrians $(\mathrm{n}=167)$, cyclists $(\mathrm{n}=148)$ and vehicle drivers $(\mathrm{n}=109)$ were surveyed on the streets and by postal means. This article characterizes the use of PMDs and the perceptions of other users. The results show a gap sometimes of great dimension between mobility needs and public awareness, as well as difficulties inherent to some traffic laws when it comes to share public spaces.

Keywords Personal mobility device $\cdot$ Pedestrian $\cdot$ Cyclist Driver $\cdot$ Roadway rule 


\section{Introduction}

Les fauteuils roulants motorisés, les triporteurs et les quadriporteurs sont classés comme des Aides à la mobilité motorisées (AMM). Les utilisateurs de ces AMM, considérés partout dans le monde comme des piétons, bénéficient cependant d'un statut particulier dans plusieurs pays. En Europe francophone (France [1], Belgique [2] et Suisse [3]) et en Europe du Nord (Pays-Bas, Danemark et Scandinavie [4-5]), leur statut leur permet d'être à la fois un piéton et un cycliste. D'une part, la présence des AMM est admise sur les infrastructures piétonnières (en autant que l'usager aille à une vitesse ne mettant pas en péril la sécurité des usagers sur le trottoir) et de l'autre, elle l'est également sur la chaussée (en autant que les AMM aillent dans le même sens que les véhicules et les cyclistes).

Dans les pays Anglo-saxons, incluant le Canada et les États-Unis, ce modèle de polyvalence n'existe pas : l'utilisateur d'une AMM est soit considéré comme un piéton, soit comme un véhicule routier à part entière. Lorsque l'AMM est assimilée à un piéton, celle-ci doit, en l'absence de trottoir, circuler sur la chaussée dans le sens contraire aux véhicules et aux vélos. En Australie, en Nouvelle-Zélande et au Royaume-Uni, plutôt que d'accorder une option « cycliste » aux utilisateurs d'AMM, une règle de base fait en sorte que tout appareil motorisé pouvant atteindre la vitesse de $10 \mathrm{~km} / \mathrm{h}$ devient, «par défaut », un véhicule routier.

Le Royaume-Uni possède la plus complexe des législations recensées [5]. Elle prévoit trois catégories d'aides à la mobilité, dont deux sont motorisées. En plus des appareils manuels (dits de classe 1), une première catégorie d'AMM (dits de classe 2) est autorisée à aller uniquement sur les aires piétonnières. Ils ne peuvent excéder, de par leur conception, la vitesse de $6,4 \mathrm{~km} / \mathrm{h}(4 \mathrm{mph})$. Les appareils de la classe 3, les seuls autorisés à aller sur la chaussée, ne sont pas limités. Ils peuvent quand même emprunter le trottoir, car ils sont munis d'une clé bloquant la vitesse à $6,4 \mathrm{~km} / \mathrm{h}$, et cette clé doit être activée sur le trottoir et les aires piétonnières. Les appareils de classe 3 doivent en contrepartie répondre à la réglementation sur les véhicules routiers et être équipés en tant que tel. $\mathrm{La}$ classe 3 prévoit aussi des mécanismes de contrôle d'accès au statut d'AMM, dont l'immatriculation des appareils. Au Queensland, en Australie, on demande à l'utilisateur d'une AMM de porter sur soi une attestation d'incapacité à la marche.

En plus des difficultés à définir un seul et même statut partout dans le monde, on observe des problématiques de sécurité qui préoccupent certaines instances plus que d'autres. À Londres, un groupe de discussion a révélé les craintes des piétons plus vulnérables face aux AMM, notamment les plus âgés (moins mobiles et qui ont une moins bonne ouïe) qui peuvent être mis en danger du fait que les AMM sont inaudibles [6].

D'autres questionnent des éléments tels que le motif d'utilisation ou la capacité de conduite. Ce sujet est matière à débat dans le contexte où les AMM représentent pour certains une alternative à la voiture personnelle, pour des raisons pratiques ou économiques ou pour compenser la perte du permis de conduire, en raison de déficiences physico-motrices [7-9].

Les AMM ont des caractéristiques différentes selon l'appareil qui est considéré (Fig. 1). Bien qu'elles regroupent les fauteuils roulants et les scooters à trois et quatre roues, certains suggèrent de les distinguer [10]. Le fauteuil se rapprocherait davantage du piéton, alors que les tri- et quadriporteurs partagent certains points avec les cyclistes, notamment des vitesses plus élevées. Les AMM ont d'abord été conçues pour suppléer des incapacités ou des difficultés à la marche, et c'est encore vrai aujourd'hui. Il faut cependant admettre que la situation a considérablement évolué, les progrès technologiques conférant aujourd'hui robustesse, autonomie et vitesse qui font des AMM des substituts à la voiture en milieu urbain [11].

$\mathrm{Au}$ Québec, les AMM ne font l'objet d'aucune règle à l'intérieur du Code de la sécurité routière [12]. Devant ce vide juridique, doublé d'un manque de connaissances sur les usagers, leurs besoins et leurs habitudes, la Table québécoise de la sécurité routière (TQSR) a suggéré, dans son premier rapport de recommandations [13], qu'une étude complète soit réalisée sur la circulation des AMM. La Société de l'assurance automobile du Québec (SAAQ) qui gère les dossiers de sécurité routière relatifs aux usagers, ainsi que le ministère de la Santé et des Services sociaux du Québec (MSSS) qui octroie ces appareils via le réseau de la réadaptation, ont donc mandaté l'Institut national de santé publique du Québec (INSPQ) pour produire un avis de santé publique sur cette question. Dans cet Avis [14], considérant la sécurité de tous les usagers du réseau (utilisateurs d'AMM, piétons et cyclistes), ainsi que le besoin d'autonomie des personnes ayant des incapacités à la marche, l'INSPQ, conformément au modèle européen, recommande que les AMM soient autorisées à circuler sur les voies piétonnières, cyclables et routières, avec un encadrement visant à responsabiliser les utilisateurs pour assurer la sécurité de toutes les parties.

Dans son Avis, l'INSPQ recommande de définir dans le Code de la sécurité routière une nouvelle catégorie d'utilisateurs de la voie publique pour les AMM. Il recommande également d'édicter des règles de circulation en cohérence avec les règles actuelles s'appliquant aux piétons et aux cyclistes. La définition de la catégorie AMM proposée par l'INSPQ est la suivante : 


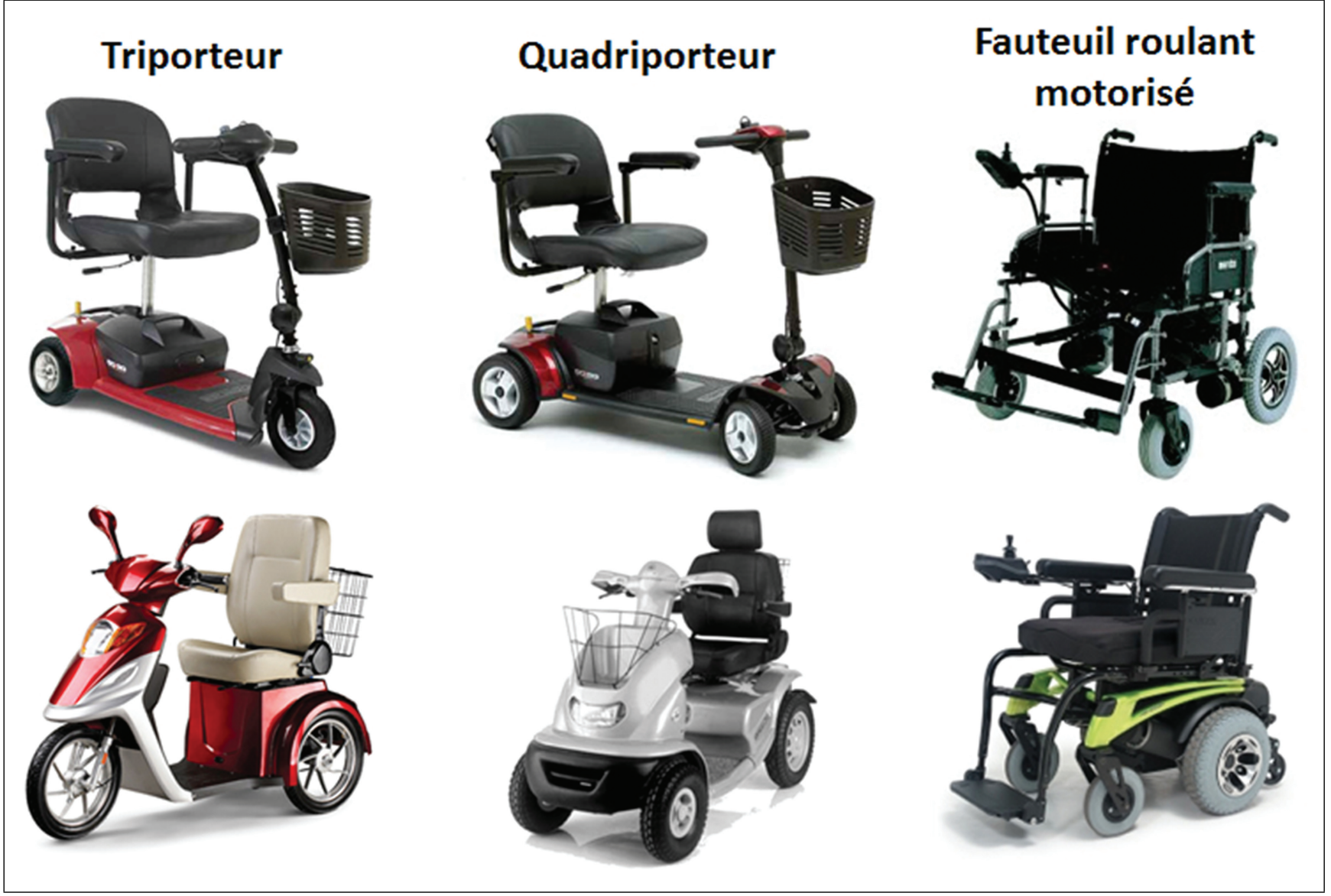

Fig. 1 Les trois types d'AMM : triporteur, quadriporteur et fauteuil roulant motorisé

Une aide à la mobilité motorisée est un appareil à un seul passager (fauteuil roulant motorisé, triporteur ou quadriporteur) conçu pour, et utilisé par, une personne ayant des incapacités à la marche restreignant sa mobilité.

Et considérant que les utilisateurs d'AMM doivent avoir un statut qui leur permet d'agir comme les piétons sur les aires piétonnières et comme les cyclistes lorsqu'ils se trouvent sur la chaussée, voici les règles de circulation proposées :

1. autoriser la circulation sur les trottoirs et les aires piétonnières ;

2. autoriser la circulation des AMM à l'extrême droite de la chaussée (à la façon d'un cycliste), si la limite affichée est $\leq 50 \mathrm{~km} / \mathrm{h}$;

3. interdire la circulation sur la chaussée en sens inverse, sauf sens unique signalé ;

4. autoriser et encourager la circulation sur les bandes et les pistes cyclables ;

5. obliger les AMM à être munies de réflecteurs à l'avant, à l'arrière, sur le côté, et d'un feu rouge à l'arrière et d'un phare blanc à l'avant en condition d'obscurité.
Pour valider ce cadre légal, un projet pilote (2011-2015) impliquant les instances municipales et gouvernementales, les forces policières et le milieu associatif a été mis sur pied. Il vise à évaluer les impacts de ces règles sur la sécurité de tous les usagers du réseau et sur l'autonomie des utilisateurs d'AMM.

Le présent article fait état de la première étape de ce projet qui a consisté à documenter les perceptions des usagers de la route (automobilistes, cyclistes et utilisateurs d'AMM) au regard de la problématique des AMM et des mesures réglementaires à envisager.

\section{Méthodologie}

Les résultats de cette enquête sont issus d'un questionnaire portant sur deux thématiques : l'usage et la perception $\mathrm{du}$ risque. Les caractéristiques de l'usage sont celles que rapportent les utilisateurs. Les habitudes des usagers ne sont pas mesurées, elles sont auto-rapportées. Quant aux perceptions, elles sont mesurées en termes de réceptivité des clientèles face à un éventuel statut pouvant être attribué aux AMM. Le questionnaire vise spécifiquement à : 
- décrire l'usage actuel des infrastructures par les utilisateurs d'AMM (seuls les déplacements hors du domicile sont considérés) ;’

- évaluer auprès des utilisateurs d'AMM, leur perception de la problématique des AMM (risque, inconfort, autonomie, mobilité) et l'acceptabilité des mesures proposées ;

- mesurer auprès des autres usagers de la route (automobilistes, cyclistes et piétons) leur perception de la problématique des AMM (risque, inconfort, autonomie, mobilité) et l'acceptabilité des mesures proposées.

\section{Type de questionnaire}

Pour répondre aux besoins de l'étude, deux formats d'enquête sont utilisés : l'entrevue en direct et le questionnaire papier auto-administré. L'entrevue en direct assure un taux de réponse optimal et rehausse la qualité des renseignements, notamment parce qu'elle augmente la probabilité que tous comprennent la question de la même façon. Le fait de recourir à des observateurs nous a aussi permis de corriger des données sur l'utilisation du réseau en temps réel. L'observateur notait, pour chaque utilisateur d'AMM interviewé, son emplacement sur la chaussée, le trottoir ou la voie cyclable, un éventuel changement d'infrastructure, ainsi que le sens de circulation. Ces précieuses données, compilées sur place par l'interviewer, permettent de faire un lien entre l'opinion du répondant et l'usage réel qu'il fait des infrastructures.

La version auto-administrée du questionnaire a été utilisée de deux façons : dans un scénario où il était prévu, et dans un autre où il a fallu y recourir pour suppléer des données manquantes. Dans le scénario prévu, le formulaire devait nécessairement être auto-administré car il s'agissait de trouver un moyen de rejoindre efficacement les automobilistes. L'option retenue a consisté à remettre le formulaire dans une enveloppe pré-affranchie aux automobilistes immobilisés dans le trafic. Dans l'autre cas, il s'agissait de suppléer la relative rareté des données concernant les utilisateurs d'AMM qui constituent le sujet principal d'étude. N'ayant qu'une centaine d'observations impliquant des AMM «dans la rue », il a été décidé de les rejoindre par le biais du milieu associatif, par téléphone et par courriel. Cette stratégie a permis de doubler le nombre de répondants AMM. Cette tactique s'est aussi avérée profitable du fait que la quasi-totalité des commentaires reçus se trouvent sur des formulaires auto-administrés. Sur le terrain, les répondants se sont montrés plus pressés et moins enclins à développer leurs idées. La validité de cette portion de l'échantillon a été estimée par une comparaison entre les réponses prises de façon aléatoire, sur le terrain, et celles issues de l'approche réalisée par le milieu associatif.

\section{Sélection des sujets}

Sur le terrain, la sélection des sujets s'est faite par l'observateur selon une procédure séquentielle différente pour chaque clientèle. Pour les utilisateurs d'AMM, les piétons et les cyclistes, les répondants sont interpellés dans cet ordre respectif. Les observations débutent par un utilisateur d'AMM, et une fois l'entrevue terminée, on invite un piéton, puis un cycliste, un utilisateur d'AMM, et ainsi de suite. Chaque catégorie d'usager est cependant traitée avec un « facteur d'approche » différent. Les utilisateurs d'AMM sont sélectionnés de façon systématique. Puisqu'ils sont beaucoup moins nombreux que les autres clientèles, tous les utilisateurs d'AMM qui arrivent vers l'observateur (à moins de $10 \mathrm{~m}$ du carré de l'intersection) sont invités à répondre au questionnaire. Quant aux piétons et aux cyclistes, l'interviewer aborde le $2^{\mathrm{e}}$ individu qui se présente vers lui. L'observateur commence la séquence de comptage dès qu'il termine avec le dernier sujet et qu'il repositionne son regard face au trafic. En cas de refus de répondre, l'observateur répète sa demande au prochain usager qui se présente à lui.

Les automobilistes ne sont pas sollicités de la même façon. L'observateur remet à l'automobiliste un questionnaire inséré dans une enveloppe pré-affranchie. Il lui explique brièvement le but de l'étude et la procédure, en demandant de compléter et de poster le questionnaire. Pour inviter l'automobiliste, l'observateur se place un peu en amont du feu de circulation ou du panneau d'arrêt, de façon à intercepter le $3^{\mathrm{e}}$ véhicule qui s'immobilise à l'intersection. Puisque cette démarche est exigeante, et qu'elle peut être réalisée plus facilement lorsque l'achalandage est moyen (heures de pointe à éviter), les automobilistes ne font pas partie de la rotation séquentielle avec les autres clientèles. Les sujets sont plutôt sélectionnés en bloc, lors d'une opération « automobilistes ».

\section{Taux de participation}

Le taux de participation est très satisfaisant. Il atteint $69 \%$ chez les piétons, $73 \%$ chez les cyclistes, et $76 \%$ chez les utilisateurs d'AMM. Les usagers ayant refusé de répondre montraient souvent des signes d'empressement. Malgré un taux de réponses moins élevé, les piétons semblent plus enclins à répondre. Les automobilistes furent probablement les plus difficiles à intéresser. Dans le feu de l'action, il s'est avéré impossible de compter les refus chez les automobilistes. Toutefois, les 109 enveloppes retournées sur les 437 distribuées confèrent un taux de retour de $25 \%$, ce qui est tout de même intéressant, compte tenu du défi. 


\section{Période et lieux de collecte}

Les données de cette enquête ont été amassées sur une trentaine de journées de semaine et de fin de semaine, échelonnées entre le 3 juin et le 24 juillet 2011. Deux observateurs se trouvaient sur les lieux, entre $10 \mathrm{~h} 00$ et $16 \mathrm{~h} 00$.

Les sites de collecte ont été identifiés dans les trois municipalités qui participent au projet pilote : Montréal, Victoriaville et Magog. Ces municipalités ont été choisies afin d'obtenir une diversité de contextes. D'autres questionnaires auto-administrés ont été recueillis à Saguenay et Sherbrooke, auprès des AMM seulement. Les points de vue exprimés par les usagers sont susceptibles de refléter cette diversité des milieux de vie. À Montréal, la plus grande métropole québécoise, les AMM doivent circuler parmi une horde de cyclistes, de piétons et d'automobilistes. Les agglomérations moins centrales sont aussi intéressantes, du fait que la population vieillissante se retire souvent des zones agitées et achalandées. Les questionnaires ont été adressés dans deux arrondissements : à Lachine, une agglomération de banlieue, et au centre-ville, dans l'arrondissement de Ville-Marie. Victoriaville est une ville de taille moyenne, ceinturée de petits hameaux, où il est intéressant d'avoir le point de vue des usagers qui circulent dans des conditions d'achalandage beaucoup moins intenses, et parfois vers des milieux plus ruraux. Quant au choix d'une municipalité de plus petite taille (Magog), il réside dans l'attrait d'un contexte touristique, où les artères principales sont tout de même très achalandées. Enfin, Sherbrooke et Saguenay sont des villes de taille moyenne qui jouent le rôle de métropole régionale.

Une attention particulière a aussi été portée au choix des 15 sites de collecte (Tableau 1). Une liste préliminaire, suggérée par les intervenants municipaux, fut validée sur le terrain. Une première visite a permis d'assurer que l'échantillon n'allait pas comporter deux sites similaires dans un même milieu de vie (municipalité). Cette présélection des sites a aussi permis de constater que chacun d'entre eux allait générer une quantité suffisante d'informations, surtout au regard des AMM. Les paramètres qui ont servi à caractériser les sites sont le nombre de voies, la présence de terre-plein, le volume de véhicules, la présence et la largeur des trottoirs, ainsi que le type de voie cyclable.

Tableau 1 Caractéristiques des sites de collecte

\begin{tabular}{|c|c|c|c|c|c|c|}
\hline Intersection & Rue Voies & Débit & $\begin{array}{l}\text { Trottoir } \\
\text { Présence }\end{array}$ & Largeur & Voie cyclable & Terre-plein \\
\hline
\end{tabular}

\section{Magog}

Bowen/Merry

Route 112 (piste pointe

Merry)

Sherbrooke/Jean-Paul II

Sherbrooke/Péladeau

Victoriaville

Bigarré/Perreault

Notre-Dame

Est-Ouest/Carignan

Sherbrooke

Belvédère/Place de la Gare
Montréal

Atwater/Saint-Ambroise

St-Joseph/32 $2^{\mathrm{e}}$ Avenue

$18^{\mathrm{e}}$ Avenue/Notre-Dame

$32^{\mathrm{e}}$ Avenue/Remembrance

Saint-Denis/Rachel

René-Lévesque/Berri

Sainte-Catherine/Berri

Mont-Royal/Parc

\section{$2 / 2$}

2

$3 / 2$

$4 / 2$

$4 / 2$

2- $4 / 2$

$4 / 2$

$2 / 2$

$2 / 1$

$2 / 2$

$6 / 2$

$4 / 2$

$6 / 4$

$2 / 4$

$4 / 4$

\section{Faible \\ Moyen}

1 côté/2 côtés

1 côté/Absent

Moyen

Moyen

2 côtés/ 1 côté

1 côté/Absent

Moyen

Absent/1 côté

Moyen

2 côtés/ 2 côtés

Élevé

2 côtés/2 côtés

Faible

Faible

Faible

2 côtés/ 2 côtés

2 côtés/ 1 côté

Moyen

2 côtés/ 2 côtés

Élevé

Élevé

2 côtés $/ 2$ côtés

2 côtés $/ 2$ côtés

2 côtés $/ 2$ côtés

Élevé

Élevé
2 côtés/ 2 côtés

2 côtés $/ 2$ côtés $\begin{array}{ll}\text { Étroit/Étroit } & \text { Bande } \\ \text { Étroit/Absent } & \text { Site propre }\end{array}$

Étroit/Étroit

Étroit/

Bande

$-$

Absent/Étroit

Site

propre/bande

Large/Étroit

Site propre

Étroit/Étroit

Large/Large

Étroit/Étroit

Étroit/Étroit

Étroit/Étroit

Large/Large

Large/Large

Bande

Site propre

Bande

$-$

Bande

Site propre/Site

propre

Large/Large

Site propre
$-$

Oui/-

Oui/-

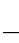

$-$

Oui/-

$-$

Oui/- 


\section{Résultats}

\section{Profil des répondants}

Quatre-vingt-quinze utilisateurs d'AMM ont complété le questionnaire auto-administré et 91 l'ont complété sur le terrain, par le biais d'une entrevue (Tableau 2). Par ailleurs, 167 piétons et 148 cyclistes ont complété le questionnaire, tous par entrevue directe. Les 109 automobilistes de l'échantillon ont complété la version auto-administrée.

L'île de Montréal regroupe $41 \%$ des répondants. La ventilation est toutefois inégale en fonction des catégories de répondants (Tableau 3). Ainsi, Montréal se démarque par l'importance de sa contribution au niveau des cyclistes, des piétons et des automobilistes, et Victoriaville, de même que Magog et Sherbrooke, ont été déterminantes pour recueillir des informations auprès des utilisateurs d'AMM.

Les données disponibles ne permettent pas d'évaluer avec précision le pourcentage d'utilisateurs d'AMM dans la population totale, et par conséquent la taille de l'échantillon par rapport au bassin d'utilisateurs d'AMM. D'une part, aucune donnée gouvernementale n'est disponible sur l'utilisation réelle des appareils octroyés, certains étant entreposés et non utilisés. De plus, les ventes libres ne sont pas comptabilisées. Steyn et al. [4] ont quant à eux estimé que le nombre d'AMM correspondrait à $10 \%$ des adultes avec des problèmes de mobilité. En appliquant cette proportion au taux moyen de personnes à mobilité réduite attendues par tranche d'âge, ils ont obtenu un taux moyen de 1,2 AMM par 1000 habitants pour la région de Fraser Valley en Colombie-Britannique. Or, si l'on applique ce même facteur dans les villes sondées, on obtiendrait près de 2000 utilisateurs d'AMM à Montréal, 200 à Sherbrooke et un peu plus de 50 à Victoriaville. Ces résultats semblent $a$ priori conservateurs, du fait qu'à Victoriaville par exemple, 68 utilisateurs d'AMM ont été sondés.

La majorité des répondants sont des hommes, mais on remarque des différences inhérentes au mode de déplacement (Tableau 4). Ainsi la proportion de femmes et d'hommes est presque équivalente chez les piétons et les automobilistes, mais les deux tiers des répondants cyclistes et utilisateurs d'AMM sont des hommes. Les écarts selon la clientèle sont beaucoup plus importants selon l'âge (Tableau 5). Si le groupe des piétons, des cyclistes et des automobilistes recueillent des parts identiques d'adultes de 45 à 65 ans (47\%-48\%), les utilisateurs d'AMM n'obtiennent que $28 \%$ dans cette tranche d'âge. Les utilisateurs d'AMM sont beaucoup plus âgés, en moyenne,

Tableau 2 Type de répondant par méthode de collecte

\begin{tabular}{|c|c|c|c|c|c|c|}
\hline \multirow[t]{2}{*}{$\begin{array}{l}\text { Type de } \\
\text { répondant }\end{array}$} & \multicolumn{2}{|c|}{$\begin{array}{l}\text { Questionné par } \\
\text { observateur (A) }\end{array}$} & \multicolumn{2}{|c|}{$\begin{array}{l}\text { Questionnaire } \\
\text { auto-administré (B) }\end{array}$} & \multicolumn{2}{|c|}{ Questionnaires A + B } \\
\hline & $\mathbf{N b}$ & $\%$ & $\mathbf{N b}$ & $\%$ & $\mathbf{N b}$ & $\%$ \\
\hline AMM & 91,0 & 22,4 & 95,0 & 46,6 & 186,0 & 30,5 \\
\hline Piéton & 167,0 & 41,1 & - & - & 167,0 & 27,4 \\
\hline Cycliste & 148,0 & 36,5 & - & - & 148,0 & 24,2 \\
\hline Automobiliste & - & - & 109,0 & 53,4 & 109,0 & 17,9 \\
\hline Total & 406,0 & 100,0 & 204,0 & 100,0 & 610,0 & 100,0 \\
\hline
\end{tabular}

Tableau 3 Provenance des répondants

\begin{tabular}{|c|c|c|c|c|c|c|c|c|c|c|}
\hline \multirow[t]{2}{*}{ Municipalité } & \multicolumn{2}{|l|}{$\mathbf{A M M}$} & \multicolumn{2}{|c|}{ Piéton } & \multicolumn{2}{|c|}{ Cycliste } & \multicolumn{2}{|l|}{ Auto } & \multicolumn{2}{|l|}{ Total } \\
\hline & $\mathbf{N b}$ & $\%$ & $\mathbf{N b}$ & $\%$ & $\mathbf{N b}$ & $\%$ & $\mathbf{N b}$ & $\%$ & $\mathbf{N b}$ & $\%$ \\
\hline Magog & 50,0 & 26,9 & 45,0 & 26,9 & 26,0 & 17,6 & 30,0 & 27,5 & 151,0 & 24,8 \\
\hline Montréal & 42,0 & 22,6 & 82,0 & 49,1 & 80,0 & 54,0 & 45,0 & 41,3 & 249,0 & 40,8 \\
\hline Saguenay & 4,0 & 2,1 & - & - & - & - & - & - & 4,0 & 0,7 \\
\hline Sherbrooke & 22,0 & 11,8 & 3,0 & 1,8 & 2,0 & 1,4 & - & - & 27,0 & 4,4 \\
\hline Victoriaville & 68,0 & 36,6 & 37,0 & 22,2 & 40,0 & 27,0 & 34,0 & 31,2 & 179,0 & 29,3 \\
\hline Total & 186,0 & 100,0 & 167,0 & 100,0 & 148,0 & 100,0 & 109,0 & 100,0 & 610,0 & 100,0 \\
\hline
\end{tabular}




\begin{tabular}{|c|c|c|c|c|c|}
\hline Genre & AMM & Piéton & Cycliste & Conducteur & Total \\
\hline Femme & 36,0 & 54,9 & 31,5 & 46,6 & 42,4 \\
\hline Homme & 64,0 & 45,1 & 68,5 & 53,4 & 57,6 \\
\hline Total & 100 & 100 & 100 & 100 & 100 \\
\hline
\end{tabular}

\begin{tabular}{|c|c|c|c|c|c|}
\hline Ville & AMM & Piéton & Cycliste & Conducteur & Total \\
\hline $18-44$ ans & 14,9 & 30,5 & 50,0 & 26,4 & 30,0 \\
\hline $45-64$ ans & 28,0 & 48,2 & 46,6 & 47,2 & 41,6 \\
\hline 65 ans et plus & 57,1 & 21,3 & 3,4 & 26,4 & 28,4 \\
\hline Total & 100 & 100 & 100 & 100 & 100 \\
\hline
\end{tabular}

que les autres catégories de répondants. Seulement $3 \%$ des cyclistes ont 65 ans et plus, alors que ce groupe est fortement majoritaire chez utilisateurs d'AMM (57\%).

\section{Caractéristiques de l'usage AMM}

La majorité des répondants utilisateurs d'AMM emploient principalement un quadriporteur lors de leurs déplacements (55\%). La part du fauteuil roulant motorisé est de $29 \%$. Très peu utilisent un triporteur (14\%), ceci s'expliquant par sa conception à trois roues, qui optimise la manœuvrabilité à l'intérieur, mais qui le rend trop versant, donc moins utilisé à l'extérieur. Trois usagers sur quatre se disent incapables de marcher $(37 \%)$ ou capables de le faire sur une très courte distance (39\%), soit moins de 100 mètres (Tableau 6). Les autres sont capables de marcher (19\%), mais sur une distance relativement limitée, généralement moins d'un kilomètre. Ceux qui se disent aptes à marcher sur une distance supérieure à un kilomètre ne représentent que $6 \%$ des utilisateurs d'AMM. Enfin, un nombre assez important d'utilisateurs de fauteuils roulants motorisés $(20 \%)$ se dit « capable de marcher un peu».

Sur le terrain, l'observateur a noté l'équipement de sécurité présent sur l'appareil. Les répondants ont aussi été questionnés, à savoir quels équipements devraient être obligatoires (Tableau 7). Les usagers ayant répondu par euxmêmes au questionnaire notaient séparément l'équipement

\begin{tabular}{|c|c|c|c|c|c|c|c|c|}
\hline \multirow[t]{2}{*}{ Capacité de déplacement } & \multicolumn{2}{|c|}{ Fauteuil motorisé } & \multicolumn{2}{|c|}{ Quadriporteur } & \multicolumn{2}{|c|}{ Triporteur } & \multicolumn{2}{|c|}{ AMM } \\
\hline & $\mathbf{N b}$ & $\%$ & $\mathbf{N b}$ & $\%$ & $\mathbf{N b}$ & $\%$ & $\mathbf{N b}$ & $\%$ \\
\hline Ne marche pas & 41 & 80,4 & 22 & 21,8 & 2 & 8,0 & 67 & 36,6 \\
\hline Marche $<100 \mathrm{~m}$ & 9 & 17,6 & 46 & 45,5 & 15 & 60,0 & 71 & 38,8 \\
\hline Marche $<1000 \mathrm{~m}$ & 1 & 2,0 & 27 & 26,7 & 4 & 16,0 & 34 & 18,6 \\
\hline Marche $>1000 \mathrm{~m}$ & - & - & 6 & 5,9 & 4 & 16,0 & 11 & 6,0 \\
\hline Total & 51 & 100 & 101 & 100 & 25 & 100 & 183 & 100 \\
\hline
\end{tabular}

\begin{tabular}{|c|c|c|c|c|c|c|c|c|}
\hline \multirow[t]{2}{*}{$\begin{array}{l}\text { Capacité de } \\
\text { déplacement }\end{array}$} & \multicolumn{2}{|c|}{ Fauteuil motorisé } & \multicolumn{2}{|c|}{ Quadriporteur } & \multicolumn{2}{|c|}{ Triporteur } & \multicolumn{2}{|l|}{ AMM } \\
\hline & Présent & Loi & Présent & Loi & Présent & Loi & Présent & Loi \\
\hline Fanion & 32,1 & 60,4 & 78,6 & 87,1 & 61,5 & 74,1 & 62,5 & 77,3 \\
\hline Réflecteurs & 69,8 & 88,7 & 84,5 & 90,1 & 92,3 & 88,9 & 81,0 & 89,5 \\
\hline Phares & 47,2 & 66,0 & 87,4 & 95,0 & 88,5 & 92,6 & 75,5 & 86,2 \\
\hline Klaxon & 71,7 & 75,5 & 91,3 & 96,0 & 96,2 & 92,6 & 85,9 & 89,5 \\
\hline Feux de direction & 9,4 & 39,6 & 89,1 & 88,1 & 96,3 & 92,6 & 66,9 & 74,6 \\
\hline Rétroviseur & 17,0 & 41,5 & 85,4 & 96,0 & 88,5 & 88,9 & 65,8 & 79,0 \\
\hline $\begin{array}{l}\text { Aucun } \\
\text { équipement }\end{array}$ & 3,8 & 1,9 & 1,0 & 2,0 & 3,8 & 3,7 & 3,3 & 2,2 \\
\hline
\end{tabular}


présent sur leur appareil et celui devant faire l'objet d'une réglementation. Les usagers d'AMM sont très nombreux à estimer que les réflecteurs $(90 \%)$, les phares $(86 \%)$ et le klaxon (90\%) devraient faire l'objet d'une réglementation. En fait, la majorité des usagers possèdent déjà ces équipements ( $76 \%$ à $86 \%$ ), hormis les fauteuils roulants motorisés. Le rétroviseur, le fanion et les feux de direction obtiennent eux aussi un fort taux de désirabilité d'une loi à leur égard. Un des faits saillants est l'intérêt marqué des usagers d'AMM à normaliser les équipements qu'ils possèdent déjà. Plusieurs considèrent même qu'il faudrait rendre obligatoire certains équipements qu'ils n'ont pas encore. Ainsi, les usagers d'AMM semblent bien enclins à ce qu'un équipement minimal soit exigé, ceux faisant l'objet du plus large consensus étant le trio phares-réflecteurs-klaxon. Il faut enfin souligner le manque d'équipements et la difficulté de les installer sur les fauteuils roulants motorisés. Ceci n'empêche pas leurs utilisateurs de reconnaître l'importance de ce type d'équipement puisque la proportion de ceux qui sont en faveur de la loi peut être de deux à trois fois plus élevée que ce qui est observé sur les appareils.

Des 186 utilisateurs d'AMM qui ont été sondés, $70 \%$ disaient connaître $(\mathrm{n}=131)$ la vitesse maximale de leur appareil (Fig. 2), généralement parce qu'il est muni d'un odomètre. Selon les répondants, un appareil sur six (17\%) ne peut excéder $10 \mathrm{~km} / \mathrm{h}$. La très grande majorité des appareils $(2 / 3)$ peuvent circuler à une vitesse allant de 10 à $19 \mathrm{~km} / \mathrm{h}(65 \%)$ et $18 \%$ de répondants affirment circuler sur un appareil pouvant aller à plus de $20 \mathrm{~km} / \mathrm{h}$, ceux-ci étant tous des tri- ou quadriporteurs. La quasi-totalité des fauteuils roulants motorisés ne peuvent pas excéder $15 \mathrm{~km} / \mathrm{h}$ (94\%).

La vitesse maximale potentielle est une donnée déterminante pour certains, qui peut cependant être distinguée de la vitesse réellement pratiquée. Les utilisateurs d'AMM ont fourni des indications quant à la vitesse de croisière généralement pratiquée sur les trois principaux types d'infrastructures. Ces données ont été agrégées afin de simplifier l'illustration (Fig. 3). Même si très peu d'AMM ont une vitesse limitée à moins de $10 \mathrm{~km} / \mathrm{h}(17 \%)$, à la

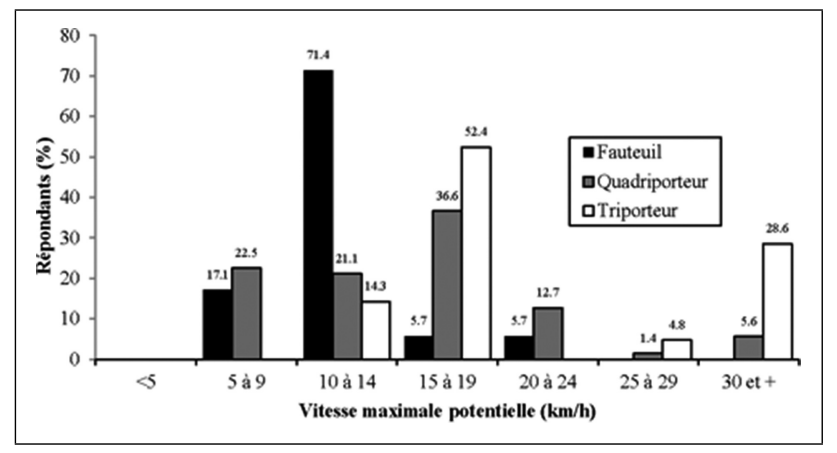

Fig. 2 Vitesse maximale de l'AMM estimée par son utilisateur

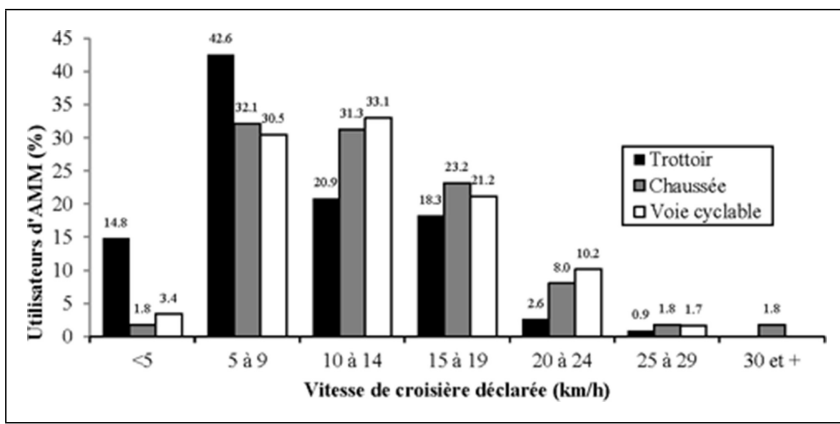

Fig. 3 Vitesse de croisière déclarée par le répondant selon l'infrastructure

conception ou par bridage, sur le trottoir, c'est une majorité d'utilisateurs qui dit circuler à une vitesse inférieure à $10 \mathrm{~km} / \mathrm{h}(57 \%)$. À noter cependant que $43 \%$ des utilisateurs d'AMM affirment circuler sur le trottoir à une vitesse supérieure à $10 \mathrm{~km} / \mathrm{h}$. Sur la chaussée ou les voies cyclables, deux utilisateurs sur trois $(66 \%)$ disent aller en moyenne à plus de $10 \mathrm{~km} / \mathrm{h}$. La moyenne des vitesses de croisière déclarées par les utilisateurs d'AMM équivaut à $8,9 \mathrm{~km} / \mathrm{h}$ sur le trottoir, comparativement à $12,3 \mathrm{~km} / \mathrm{h}$ et $11,9 \mathrm{~km} / \mathrm{h}$ sur la chaussée et la voie cyclable respectivement. La vitesse sur le trottoir semble donc un peu élevée en comparaison avec la vitesse normale de marche d'un piéton, qui se situe autour de $5 \mathrm{~km} / \mathrm{h}$ [15].

Au Québec, l'usage hivernal est une question importante car le climat nordique rend souvent la pratique difficile [16]. Les répondants mentionnent que le transport adapté est beaucoup sollicité l'hiver, car il est très dangereux de circuler dans les rues. Certains mentionnent que les gens mettent leur neige sur le trottoir, même lorsque ce dernier vient tout juste d'être déneigé. L'hiver, la moitié des utilisateurs d'AMM ne sortent pas (Tableau 8). Mais il y a tout de même un répondant sur trois qui dit défier les rigueurs hivernales (34\%). Les plus aguerris sont de loin les utilisateurs de fauteuils roulants motorisés. Parmi eux, un sur six ne sort jamais l'hiver (17\%), alors qu'ils sont $58 \%$ à circuler en tout temps ou assez fréquemment, car ils disent " ne pas avoir le choix », puisque c'est leur seul moyen de déplacement. Et même muni d'un véhicule adapté pour se déplacer, l'utilisateur d'un fauteuil doit tout de même rejoindre son fauteuil une fois à l'extérieur du véhicule.

Les utilisateurs d'AMM choisissent avec soin la période du jour pour effectuer leurs déplacements (Tableau 9). Ils sortent surtout le matin $(65 \%)$ et l'après-midi $(87 \%)$, un peu moins en soirée (37\%).

Bien qu'aucune question n'adresse directement l'enjeu de l'achalandage urbain, les usagers disent éviter les endroits achalandés et les axes priorisés aux heures de pointe, préférant ne pas s'exposer à la circulation véhiculaire. Les utilisateurs d'AMM circulent très rarement la nuit, surtout ceux qui se promènent en fauteuil, car il n'y a pas de phares 


\begin{tabular}{|c|c|c|c|c|c|c|c|c|}
\hline \multirow[t]{2}{*}{ Usage hivernal de l'AMM } & \multicolumn{2}{|c|}{ Fauteuil motorisé } & \multicolumn{2}{|c|}{ Quadriporteur } & \multicolumn{2}{|c|}{ Triporteur } & \multicolumn{2}{|c|}{ AMM } \\
\hline & $\mathbf{N b}$ & $\%$ & $\mathbf{N b}$ & $\%$ & $\mathbf{N b}$ & $\%$ & $\mathbf{N b}$ & $\%$ \\
\hline Jamais & 9 & 17,3 & 63 & 61,8 & 17 & 65,4 & 92 & 50,0 \\
\hline Rarement & 13 & 25,0 & 13 & 12,7 & 4 & 15,4 & 30 & 16,3 \\
\hline Assez fréquemment & 13 & 25,0 & 12 & 11,8 & 3 & 11,5 & 29 & 15,8 \\
\hline Toujours & 17 & 32,7 & 14 & 13,7 & 2 & 7,7 & 33 & 17,9 \\
\hline Total & 52 & 100 & 102 & 100 & 26 & 100 & 184 & 100 \\
\hline
\end{tabular}

Tableau 9 Période d'utilisation des AMM

\begin{tabular}{|c|c|c|c|c|c|c|c|c|}
\hline \multirow[t]{2}{*}{ Période d'utilisation des AMM } & \multicolumn{2}{|c|}{ Fauteuil motorisé } & \multicolumn{2}{|c|}{ Quadriporteur } & \multicolumn{2}{|c|}{ Triporteur } & \multicolumn{2}{|c|}{ AMM } \\
\hline & $\mathbf{N b}$ & $\%$ & $\mathbf{N b}$ & $\%$ & $\mathbf{N b}$ & $\%$ & $\mathbf{N b}$ & $\%$ \\
\hline Matin & 26 & 49,1 & 63 & 61,2 & 18 & 69,2 & 110 & 65,9 \\
\hline Après-midi & 36 & 67,9 & 82 & 79,6 & 24 & 92,3 & 145 & 86,8 \\
\hline Soirée & 20 & 37,7 & 30 & 29,1 & 9 & 34,6 & 62 & 37,1 \\
\hline Nuit & - & - & 4 & 3,9 & 1 & 3,8 & 5 & 3,0 \\
\hline Heures de pointe & 5 & 9,4 & 8 & 7,8 & 3 & 11,5 & 17 & 10,2 \\
\hline À toute heure & 19 & 35,8 & 20 & 19,4 & 1 & 3,8 & 42 & 25,1 \\
\hline
\end{tabular}

sur l'appareil. La principale distinction tient au fait que les utilisateurs de fauteuil sont plus du tiers à circuler « à toute heure du jour » $(35 \%)$, alors que cette proportion atteint $19 \%$ chez les utilisateurs de quadriporteur, et seulement $4 \%$ chez ceux qui emploient un triporteur.

L'utilisation des trottoirs est une question fondamentale. Peu importe qu'il soit large (Fig. 4) ou étroit (Fig. 5), il a été demandé aux répondants de dire à quelle fréquence ils utilisent le trottoir, lorsqu'il y en a un. Les réponses témoignent de deux pratiques différentes, qui divisent les usagers en deux grands groupes (Tableau 10). Un peu moins de la moitié disent y aller rarement ou jamais, alors qu'une légère majorité y va toujours (18\%) ou assez fréquemment (36\%). La réticence à emprunter le trottoir est un peu plus marquée chez les utilisateurs de quadriporteur.

Les répondants mentionnent que l'utilisation du trottoir dépend de plusieurs facteurs. D'abord de ses caractéristiques physiques et de sa largeur, mais aussi à cause des bordures dénivelées et des bateaux pavés (arasement de trottoir). Ces aménagements sont nécessaires pour accéder à l'infrastructure, car elle est surélevée par rapport à la chaussée, mais les pentes prononcées et l'angle de ces pentes affectent la stabilité de l'appareil, notamment en fauteuil et en triporteur, et augmentent le risque de chute. D'autres ont mentionné que les bandes tactiles pour les personnes non voyantes affectent parfois la stabilité de l'appareil.

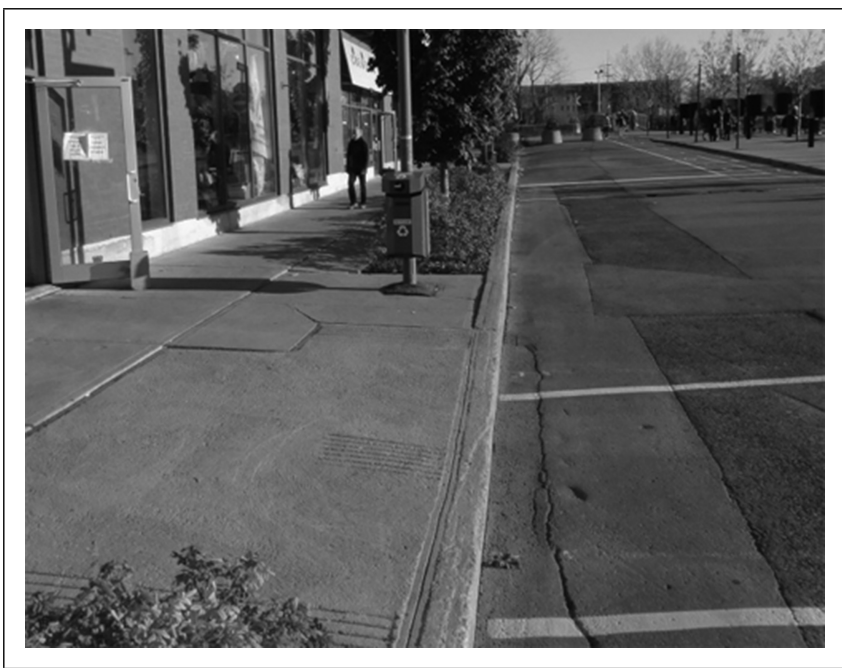

Fig. 4 Trottoir large à Montréal

Plusieurs ont dit éprouver des malaises à utiliser le trottoir lorsqu'il est achalandé, surtout lorsqu'ils sont étroits. Dans ce cas, les piétons se sentent pratiquement obligés de descendre dans la rue lorsqu'ils rencontrent une AMM. Et à l'inverse, plusieurs usagers qui utilisent le trottoir disent le faire en tout temps parce qu'ils craignent les véhicules, ou occasionnellement lorsqu'il y en a trop sur 


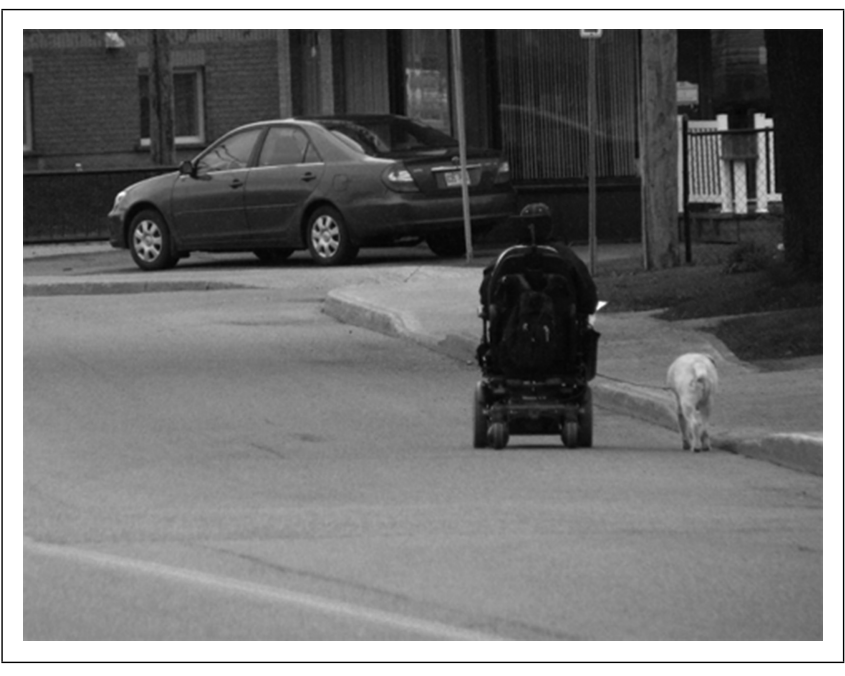

Fig. 5 Trottoir étroit à Victoriaville

la chaussée. L'achalandage sur le trottoir et la sur la chaussée a donc une influence sur le choix de l'infrastructure empruntée.

Les utilisateurs d'AMM boudent jusqu'à un certain point le trottoir mais qu'en est-il de la sécurité sur la chaussée ? On entend souvent dire, à tort ou à raison, que celle-ci est plus dangereuse, en citant la présence de véhicules. Mais est-ce suffisant pour décourager cette pratique ? Les résultats font état d'une polarisation en deux grands groupes. À la question : « dans quelle proportion utilisez-vous la chaussée, plutôt que les autres infrastructures ? », $44 \%$ des utilisateurs d'AMM disent l'utiliser rarement ou jamais, alors que $56 \%$ disent le faire toujours ou assez fréquemment (Tableau 11). Cette proportion est un peu plus importante chez les utilisateurs de fauteuil motorisé $(61 \%)$. Toutefois, la part des utilisateurs de fauteuil qui le font systématiquement (8\%) est moins élevée que celle des quadriporteurs $(17 \%)$ et des triporteurs $(19 \%)$. Mis à part cette nuance, la perception des utilisateurs est sensiblement la même peu importe l'appareil utilisé.

Les répondants qui préfèrent la chaussée justifient généralement leur choix sur l'état des trottoirs. Certains signalent qu'ils utilisent la chaussée car il y a moins de risque de blessure pour le dos que sur les trottoirs. D'autres affirment qu'il est plus sécuritaire pour l'AMM et les piétons que l'AMM circule sur la chaussée. Tout cela dépend bien entendu des trajets que ces derniers ont à effectuer. Par ailleurs, l'utilisation de la chaussée pourrait aussi être en partie liée à l'offre déficitaire et sporadique de trottoirs. Parfois placé d'un seul côté de la rue, parfois des deux, le trottoir est presque toujours absent des secteurs résidentiels à faible ou moyenne densité d'habitation. Ceci pourrait expliquer la propension des usagers québécois à adopter la chaussée, de façon générale, plutôt que le trottoir. Enfin, l'absence fréquente de trottoir a une incidence sur le cadre légal car les règles de circulation à élaborer doivent tenir

\begin{tabular}{|c|c|c|c|c|c|c|c|c|}
\hline \multirow[t]{2}{*}{ Fréquence d'utilisation du trottoir } & \multicolumn{2}{|c|}{$\underline{\text { Fauteuil motorisé }}$} & \multicolumn{2}{|c|}{ Quadriporteur } & \multicolumn{2}{|c|}{ Triporteur } & \multicolumn{2}{|c|}{$\underline{\mathbf{A M M}}$} \\
\hline & $\mathbf{N b}$ & $\%$ & $\mathbf{N b}$ & $\%$ & $\mathbf{N b}$ & $\%$ & $\mathbf{N b}$ & $\%$ \\
\hline Jamais & 4 & 7,5 & 21 & 21,0 & 4 & 15,4 & 30 & 16,4 \\
\hline Rarement & 18 & 34,0 & 31 & 31,0 & 7 & 26,9 & 56 & 30,6 \\
\hline Assez fréquemment & 21 & 39,6 & 30 & 30,0 & 12 & 46,2 & 65 & 35,5 \\
\hline Toujours & 10 & 18,9 & 18 & 18,0 & 3 & 11,5 & 32 & 17,5 \\
\hline Total & 53 & 100 & 100 & 100 & 26 & 100 & 183 & 100 \\
\hline
\end{tabular}

Tableau 11 Fréquence de l'utilisation de la chaussée vs autres infrastructures

\begin{tabular}{|c|c|c|c|c|c|c|c|c|}
\hline \multirow[t]{2}{*}{ Fréquence d'utilisation de la chaussée } & \multicolumn{2}{|c|}{$\underline{\text { Fauteuil motorisé }}$} & \multicolumn{2}{|c|}{ Quadriporteur } & \multicolumn{2}{|c|}{$\underline{\text { Triporteur }}$} & \multicolumn{2}{|c|}{$\underline{\mathbf{A M M}}$} \\
\hline & $\mathbf{N b}$ & $\%$ & $\mathbf{N b}$ & $\%$ & $\mathbf{N b}$ & $\%$ & $\mathbf{N b}$ & $\%$ \\
\hline Jamais & 1 & 2,0 & 6 & 6,0 & 2 & 7,7 & 9 & 5,0 \\
\hline Rarement & 19 & 37,3 & 39 & 38,6 & 11 & 42,3 & 69 & 38,8 \\
\hline Assez fréquemment & 27 & 52,9 & 39 & 38,6 & 8 & 30,8 & 74 & 41,6 \\
\hline Toujours & 4 & 7,8 & 17 & 16,8 & 5 & 19,2 & 26 & 14,6 \\
\hline Total & 51 & 100 & 101 & 100 & 26 & 100 & 178 & 100 \\
\hline
\end{tabular}


compte de l'absence d'alternatives offertes aux utilisateurs d'AMM.

Pour ajouter une autre perspective, le tableau 12 illustre l'emplacement de l'AMM noté par l'observateur lors de son approche à l'intersection (position 1), et en la quittant (position 2). En présence d'une voie cyclable, celle-ci est presque toujours employée. Les principaux types de voies cyclables au Québec sont les pistes cyclables, isolées du trafic (Fig. 6). Les bandes cyclables unidirectionnelles (Fig. 7) sont très rares. La plupart des bandes cyclables sont bidirectionnelles et contiguës à la chaussée. Généralement séparées de la voie de circulation par des délinéateurs verticaux (Fig. 8), certaines sont munies d'une bordure bétonnée (Fig. 9).

Par ailleurs, la part des segments roulés sur le trottoir et la chaussée est exactement 50-50 $(\mathrm{n}=21$ sur chaque infrastructure). Enfin, malgré le faible nombre d'observations, il a été constaté que près des trois quarts des utilisateurs d'AMM roulent dans le même sens que les véhicules.

\begin{tabular}{|c|c|c|c|c|c|c|}
\hline \multirow[t]{2}{*}{ Infrastructure empruntée } & \multicolumn{2}{|c|}{ Position 1} & \multicolumn{2}{|c|}{ Position 2} & \multicolumn{2}{|c|}{ Total } \\
\hline & Nb & $\%$ & $\%$ & $\%$ & $\%$ & $\%$ \\
\hline Voie cyclable & 65 & 76,5 & 62 & 73,8 & 127 & 75,1 \\
\hline Trottoir & 8 & 9,4 & 13 & 15,5 & 21 & 12,4 \\
\hline \multicolumn{7}{|l|}{ Chaussée } \\
\hline Même sens & 9 & 10,6 & 6 & 7,1 & 15 & 8,9 \\
\hline Contresens & 3 & 3,5 & 3 & 3,6 & 6 & 3,6 \\
\hline Total & 85 & 100,0 & 84 & 100,0 & 169 & 100,0 \\
\hline
\end{tabular}

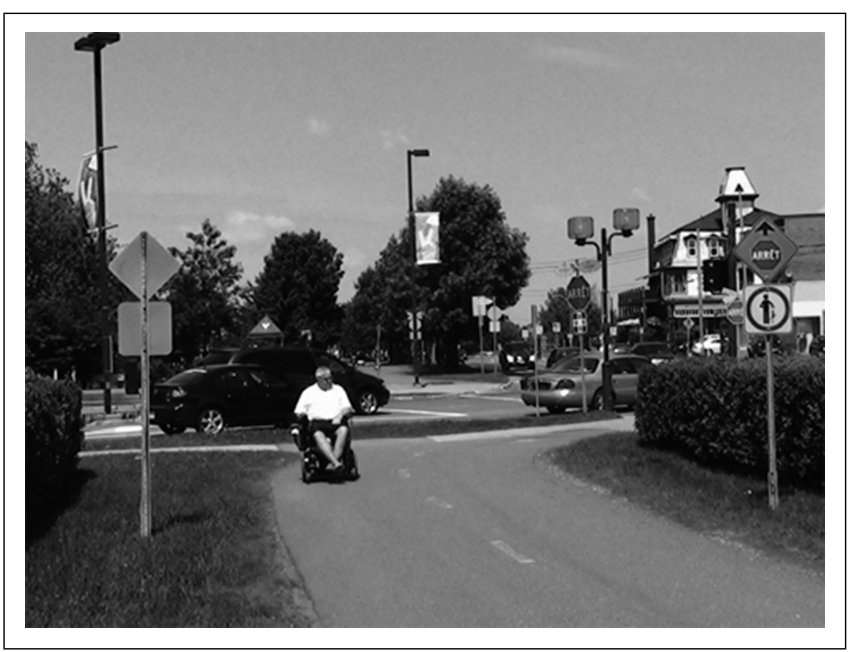

Fig. 6 Piste cyclable isolée

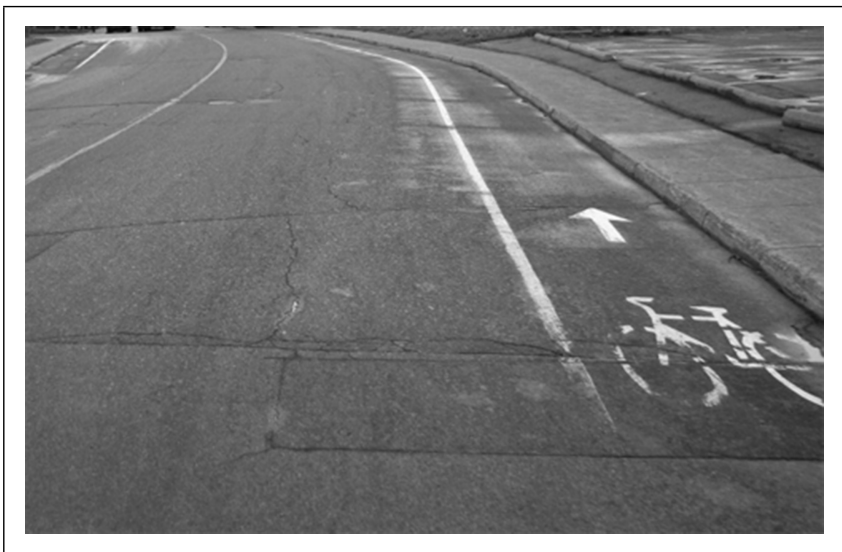

Fig. 7 Bande cyclable unidirectionnelle

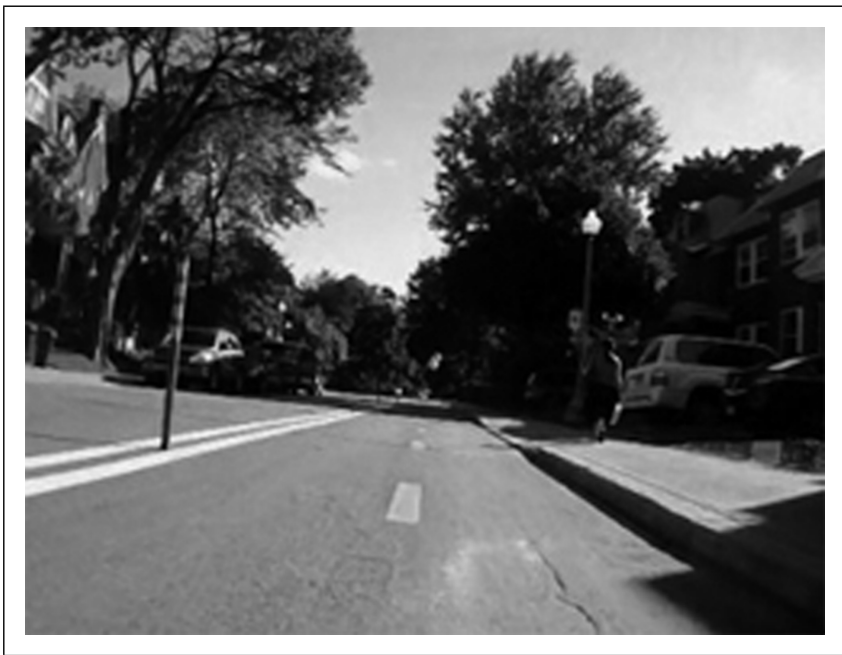

Fig. 8 Bidirectionnelle avec délinéateurs

Le dernier bloc de questions relatives à l'usage des infrastructures portait sur le milieu rural et les zones à haute vitesse. Les répondants devaient dire si en dehors des villes et en l'absence de trottoir, ils circulent sur des routes affichant $70 \mathrm{~km} / \mathrm{h}$ et plus. Un usager sur quatre $(24 \%)$ dit se déplacer sur le réseau à haute vitesse (Tableau 13). Les proportions globales se ressemblent beaucoup, mais de légères nuances sont observées en fonction de l'appareil utilisé. Ainsi, la circulation à l'extérieur des villes, sur des routes à haute vitesse, est une pratique qui est assez fréquente chez les utilisateurs de quadriporteur $(6 \%)$ et de triporteur (4\%), mais rare chez les utilisateurs de fauteuil motorisé.

Parmi ceux qui ont déclaré se rendre sur des routes rurales à $\geq 70 \mathrm{~km} / \mathrm{h}$ avec leur AMM, la très grande majorité le fait sur des accotements asphaltés (84\%) ou composés de gravier (14\%) (Tableau 14). Les pourcentages ne sont pas mutuellement exclusifs puisque plus d'un choix de réponse était possible. Toutefois, un nombre assez élevé de 


\begin{tabular}{|c|c|c|c|c|c|c|c|c|}
\hline \multirow[t]{2}{*}{ Fréquence d'utilisation des routes $\geq 70 \mathrm{~km} / \mathrm{h}$} & \multirow{2}{*}{$\begin{array}{l}\text { Fauteuil } \\
\mathrm{Nb}\end{array}$} & motorisé & \multicolumn{2}{|c|}{ Quadriporteur } & \multicolumn{2}{|c|}{ Triporteur } & \multicolumn{2}{|c|}{$\underline{\mathbf{A M M}}$} \\
\hline & & $\%$ & $\mathbf{N b}$ & $\%$ & $\mathbf{N b}$ & $\%$ & $\mathbf{N b}$ & $\%$ \\
\hline Jamais & 41 & 77,4 & 78 & 77,2 & 18 & 69,2 & 137 & 76,1 \\
\hline Rarement & 12 & 22,6 & 17 & 16,8 & 7 & 26,9 & 36 & 20,0 \\
\hline Assez fréquemment & - & - & 6 & 5,9 & 1 & 3,8 & 7 & 3,9 \\
\hline Toujours & - & - & - & - & - & - & - & - \\
\hline Total & 53 & 100 & 101 & 100 & 25 & 100 & 180 & 100 \\
\hline
\end{tabular}

\begin{tabular}{|c|c|c|c|c|c|c|c|c|}
\hline \multirow{2}{*}{ Endroit choisi sur une route $\geq 70 \mathrm{~km} / \mathrm{h}$} & \multicolumn{2}{|c|}{ Fauteuil motorisé } & \multicolumn{2}{|c|}{ Quadriporteur } & \multicolumn{2}{|c|}{$\underline{\text { Triporteur }}$} & \multicolumn{2}{|c|}{$\underline{\mathbf{A M M}}$} \\
\hline & $\mathbf{N b}$ & $\%$ & $\mathbf{N b}$ & $\%$ & $\mathbf{N b}$ & $\%$ & $\mathbf{N b}$ & $\%$ \\
\hline Chaussée & 3 & 25,0 & 7 & 30,4 & 1 & 12,5 & 11 & 25,6 \\
\hline Accotement pavé & 11 & 91,7 & 20 & 87,0 & 5 & 62,5 & 36 & 83,7 \\
\hline Accotement gravelé & 3 & 25,0 & 2 & 8,7 & 1 & 12,5 & 6 & 14,0 \\
\hline Un peu partout & 2 & 16,7 & 5 & 21,7 & 1 & 12,5 & 8 & 18,6 \\
\hline Total & 12 & 100 & 23 & 100 & 8 & 100 & 43 & 100 \\
\hline
\end{tabular}

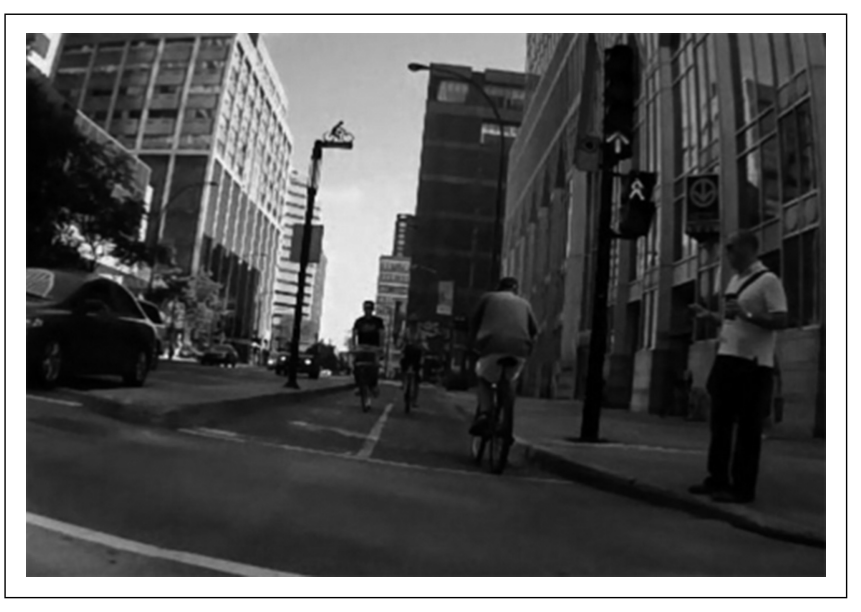

Fig. 9 Bidirectionnelle avec bordure bétonnée

répondants a dit circuler directement sur la chaussée (26\%) ou se promener un peu partout (19\%).

Sur les routes à $70 \mathrm{~km} / \mathrm{h}$ ou plus, ceux qui circulent sur la chaussée ou un peu partout posent un problème de sécurité relative. On peut ainsi se demander s'il s'agit de la voie de circulation des routes achalandées ou de routes rurales entièrement gravelées, où le volume de véhicules est très faible. Le tableau 15 illustre la distribution des conditions jugées nécessaires par les utilisateurs d'AMM pour qu'ils circulent sur de telles routes.
L'ensemble de ces données croisées confirment que les gens qui se rendent en lieu rural le font en quasi-totalité dans des conditions qui sous-tendent une prise de décision et des facteurs liés à la sécurité. Effectivement, la majorité des utilisateurs d'AMM qui vont en milieu rural le font lorsque l'accotement est assez large (86\%) et/ou pavé (74\%) et lorsque la distance à parcourir est courte $(67 \%)$. Un peu plus de la moitié identifie aussi le fait que la route doit être faiblement achalandée (56\%). Bien que le nombre d'unités d'observation par types d'appareil soit très limité, on peut voir que pour les utilisateurs de fauteuil roulant motorisé, la présence d'un accotement large et asphalté est un prérequis pour circuler en milieu rural.

\section{Opinions sur la réglementation}

Cette section présente les résultats qui portent sur la perception qu'ont les utilisateurs d'AMM, les piétons, les cyclistes et les automobilistes des règles de circulation qui pourraient être introduites et s'appliquer à l'endroit des utilisateurs d'AMM. La première question, très générale, vise à comprendre la perception des différents usagers de la route quant au statut auquel devraient être assimilés les utilisateurs d'AMM : un piéton, un cycliste, les deux, ou encore un automobiliste ? Près de $40 \%$ des répondants assimilent les utilisateurs d'AMM autant à un piéton qu'à un cycliste (Tableau 16). Si on ventile ces résultats selon la 


\begin{tabular}{|c|c|c|c|c|c|c|c|c|}
\hline \multirow[t]{2}{*}{ Condition pour aller sur une route $\geq 70 \mathrm{~km} / \mathrm{h}$} & \multicolumn{2}{|c|}{ Fauteuil motorisé } & \multicolumn{2}{|c|}{ Quadriporteur } & \multicolumn{2}{|c|}{ Triporteur } & \multicolumn{2}{|c|}{$\underline{\mathbf{A M M}}$} \\
\hline & $\mathbf{N b}$ & $\%$ & $\mathbf{N b}$ & $\%$ & $\mathbf{N b}$ & $\%$ & $\mathbf{N b}$ & $\%$ \\
\hline Route munie d'un accotement pavé & 11 & 91,7 & 15 & 65,2 & 6 & 75,0 & 32 & 74,4 \\
\hline Route munie d'un accotement large & 12 & 100,0 & 19 & 82,6 & 6 & 75,0 & 37 & 86,0 \\
\hline Route doit être peu achalandée & 9 & 75,0 & 9 & 39,1 & 6 & 75,0 & 24 & 55,8 \\
\hline Je suis accompagné(e) & 2 & 16,7 & 3 & 13,0 & 1 & 12,5 & 6 & 14,0 \\
\hline Si la distance à parcourir est courte & 9 & 75,0 & 17 & 73,9 & 3 & 37,5 & 29 & 67,4 \\
\hline Total & 12 & 100 & 23 & 100 & 8 & 100 & 43 & 100 \\
\hline
\end{tabular}

Tableau 16 Statut auquel devraient être assimilés les utilisateurs d'AMM

\begin{tabular}{|c|c|c|c|c|c|}
\hline Statut de : & АMM & Piéton & Cycliste & Auto & Total \\
\hline Piéton seulement & 22,5 & 40,6 & 45,1 & 28,7 & 33,7 \\
\hline Cycliste seulement & 18,0 & 29,4 & 15,3 & 16,7 & 20,1 \\
\hline Piéton et cycliste & 44,0 & 23,8 & 38,2 & 51,9 & 38,7 \\
\hline Automobiliste & 11,0 & 5,6 & 0,7 & 1,9 & 5,6 \\
\hline Autre & 4,5 & 0,6 & 0,7 & 0,9 & 2,0 \\
\hline Total & 100 & 100 & 100 & 100 & 100 \\
\hline
\end{tabular}

catégorie de répondant, on observe que les piétons (41\%) et les cyclistes $(45 \%)$ sont plus enclins à considérer les utilisateurs d'AMM comme piétons seulement. Les utilisateurs d'AMM et les automobilistes sont plus enclins à leur attribuer un double statut, piéton-cycliste (44\% et $52 \%$ respectivement). Enfin, les utilisateurs d'AMM se démarquent un peu des autres types d'usagers en se voyant eux-mêmes comme des automobilistes dans $11 \%$ des cas.

Au regard des environnements de pratique, les répondants devaient évaluer s'il est sécuritaire pour tous les usagers de laisser les AMM circuler sur le trottoir, l'accotement, la chaussée et les voies cyclables. En plus d'être présentés en fonction des catégories de répondant, les résultats sont aussi ventilés en fonction du type d'AMM (Tableau 17). Un consensus se dégage autour de la présence des AMM sur les voies cyclables. Neuf répondants sur dix (89\%) jugent cette pratique sécuritaire. Tous s'accordent, même les cyclistes, qui sont les premiers concernés. Quant à l'utilisation du trottoir, les piétons ont sensiblement la même opinion que les utilisateurs d'AMM. Un tiers des répondants (31\%) juge que la circulation des AMM sur le trottoir n'est pas sécuritaire. À noter que plus de la moitié des utilisateurs de fauteuil roulant manuel considèrent les trottoirs comme non sécuritaires pour leur propre circulation.

L'écart de la perception entre les différentes sondées s'accroît lorsqu'il est question de la sécurité sur les accotements et la chaussée. Ainsi, $61 \%$ des utilisateurs d'AMM jugent que leur présence est sécuritaire dans les accotements, alors que cette proportion atteint $37 \%$ à $45 \%$ chez les autres clientèles interviewées. Quant à la circulation

Tableau 17 Infrastructures jugées sécuritaires à la circulation des AMM

\begin{tabular}{lrrrrrrrr}
\hline Infrastructure & F.Mo. & Quad. & Tri. & AMM & Piéton & Cyc. & Auto & Total \\
\hline Voie cyclable & 100,0 & 90,6 & 73,5 & 89,7 & 92,6 & 88,8 & 79,6 \\
Trottoir & 48,9 & 66,7 & 69,2 & 62,1 & 63,8 & 79,4 & 72,4 & 89,1 \\
Accotement & 54,0 & 64,1 & 64,0 & 61,1 & 45,0 & 37,4 & 42,7 \\
Chaussée & 28,8 & 35,5 & 32,0 & 32,9 & 8,8 & 10,5 & 14,7 \\
Moyenne & 57,9 & 64,2 & 59,7 & 61,5 & 52,6 & 54,0 & 52,4 & 55,7 \\
\hline
\end{tabular}




\begin{tabular}{|c|c|c|c|c|c|c|c|c|}
\hline Sens de circulation & F.Mo. & Quad. & Tri. & AMM & Piéton & Cyc. & Auto & Total \\
\hline Même sens & 56,7 & 55,8 & 63,6 & 57,0 & 84,6 & 42,9 & 79,5 & 57,7 \\
\hline À contresens & 36,7 & 26,9 & 36,4 & 31,2 & 15,4 & 35,7 & 18,2 & 31,9 \\
\hline Un ou l'autre & 6,7 & 17,3 & - & 11,8 & - & 21,4 & 2,3 & 10,4 \\
\hline Total & 100 & 100 & 100 & 100 & 100 & 100 & 100 & 100 \\
\hline
\end{tabular}

\begin{tabular}{|lrrrrrrrr|}
\hline \multicolumn{7}{l}{ Tableau 19 Vitesse affichée maximale pour autoriser les AMM sur la chaussée } \\
\hline Vitesse affichée & F.Mo. & Quad. & Tri. & AMM & Piéton & Cyc. & Auto & Total \\
\hline$\leq 50 \mathrm{~km} / \mathrm{h}$ & 96,3 & 84,0 & 60,0 & 80,7 & 85,7 & 85,7 & 92,3 & 84,0 \\
$\leq 70 \mathrm{~km} / \mathrm{h}$ & - & 14,0 & 26,7 & 14,9 & 7,1 & 14,3 & 5,1 & 12,2 \\
$\leq 90 \mathrm{~km} / \mathrm{h}$ & 3,7 & 2,0 & 13,3 & 4,4 & 7,1 & - & 2,6 & 3,9 \\
Total & 100 & 100 & 100 & 100 & 100 & 100 & 100 & 100 \\
\hline
\end{tabular}

sur la chaussée, les utilisateurs d'AMM sont un tiers (33\%) à juger cette pratique sécuritaire, tandis que cette proportion n'est que $9 \%$ à $15 \%$ chez les autres clientèles.

Toujours en lien avec la circulation sur la chaussée, les répondants ont eu à se prononcer sur la notion du sens de circulation. Une majorité d'usagers juge que sur la chaussée, les AMM devraient circuler dans le même sens que les véhicules routiers (Tableau 18). Ces résultats cachent toutefois une forte polarisation des opinions. Si d'une part les automobilistes et les piétons croient que la circulation des AMM sur la chaussée devrait se faire dans le même sens que les véhicules routiers ( $80 \%$ et $85 \%$ respectivement), les utilisateurs d'AMM (57\%) et les cyclistes (43\%) sont dans l'ensemble moins favorables à cette règle.

À l'égard du type de route, les répondants devaient mentionner jusqu'à quelle limite de vitesse affichée ${ }^{1}$ la présence des AMM est sécuritaire (Tableau 19). À $84 \%$, les répondants notent qu'une AMM ne devrait pas circuler sur une route où la vitesse affichée excède $50 \mathrm{~km} / \mathrm{h}$. Mais encore ici, la ventilation présente certaines distinctions entre les catégories de répondants. Les automobilistes et les utilisateurs de fauteuil roulant motorisé sont plus conservateurs que les autres clientèles. Ils sont respectivement $4 \%$ et $8 \%$ à croire que c'est sécuritaire de laisser les AMM circuler sur une route affichant des vitesses supérieures à $50 \mathrm{~km} / \mathrm{h}$, alors que cette part est beaucoup plus importante chez les piétons,

\footnotetext{
${ }^{1}$ Pour le projet pilote, les limites de 30,40 et $50 \mathrm{~km} / \mathrm{h}$ sont incluses dans la catégorie $\leq 50 \mathrm{~km} / \mathrm{h}$, car les limites inférieures à $50 \mathrm{~km} / \mathrm{h}$ sont encore peu courantes au Québec en milieu urbain, et que $50 \mathrm{~km} / \mathrm{h}$ est encore la limite par défaut de la grande majorité des secteurs résidentiels québécois.
}

les cyclistes et les utilisateurs de quadriporteur, mais surtout, chez les utilisateurs de triporteur (40\%).

En ce qui concerne les conflits de cohabitation, une majorité croit qu'ils n'existent pas. Or, il y a tout de même $38 \%$ des répondants qui identifient l'existence de situations conflictuelles entre les utilisateurs d'AMM et les autres clientèles du réseau routier (Tableau 20). Les piétons semblent un peu moins portés à voir cette problématique, qui est surtout perçue par les utilisateurs de fauteuil roulant motorisé ( $48 \%$ ), et davantage par les automobilistes ( $55 \%$ ).

Ceux qui reconnaissent l'existence de conflits de cohabitation devaient spécifier avec quelle clientèle. À cette question, plus d'un choix était possible. Plus fréquemment, les conflits impliquent des automobiles (37\%), mais assez souvent un piéton $(23 \%)$ ou un cycliste $(21 \%)$. Les conflits avec les patineurs ou les autres utilisateurs d'AMM sont moins souvent rapportés.

La ventilation par types de clientèle permet d'ajouter de distinguer la façon dont les autres clientèles perçoivent les AMM. Ainsi, les conflits avec les piétons sont plus souvent perçus par des piétons ( $38 \%$ ), et les conflits avec les cyclistes, perçus par des cyclistes (29\%). Autre constat important, les utilisateurs de fauteuil roulant motorisé et de quadriporteur sont très préoccupés, pour leur part, par les conflits avec des automobilistes ( $43 \%$ et $44 \%$ ).

Pour expliquer la nature des conflits, plusieurs répondants expliquent que les cyclistes roulent très vite sur les voies cyclables et qu'ils ont très peur, surtout face aux patineurs et aux cyclistes de haute performance. Certains mentionnent qu'il y a aussi beaucoup de gens, et beaucoup d'autres types d'utilisateurs (ex. : marcheurs, patineurs) et qu'il est difficile de s'y frayer un chemin. 


\begin{tabular}{|c|c|c|c|c|c|c|c|c|}
\hline Conflit avec une AMM? & F.Mo. & Quad. & Tri. & AMM & Piéton & Cyc. & Auto & Total \\
\hline Oui & 48,1 & 35,0 & 42,3 & 38,0 & 31,1 & 35,1 & 55,2 & 38,4 \\
\hline \multicolumn{9}{|l|}{ Avec : } \\
\hline Piéton & 16,7 & 20,0 & 21,1 & 16,1 & 38,0 & 25,0 & 19,6 & 23,4 \\
\hline Cycliste & 19,0 & 18,2 & 26,3 & 20,2 & 12,7 & 28,6 & 21,4 & 21,4 \\
\hline Auto & 42,9 & 43,6 & 26,3 & 33,9 & 39,4 & 32,1 & 39,3 & 36,7 \\
\hline Autre AMM & - & 1,8 & 21,1 & 9,7 & 4,2 & 6,0 & 4,5 & 6,5 \\
\hline Patineur & 21,4 & 16,4 & - & 13,7 & 5,6 & 8,3 & 15,2 & 11,7 \\
\hline Autre & - & - & 5,3 & 6,5 & - & - & - & 0,3 \\
\hline
\end{tabular}

Tableau 21 Nécessité de spécifier une vitesse autorisée pour les AMM

\begin{tabular}{|c|c|c|c|c|c|c|c|c|}
\hline Infrastructure & F.Mo. & Quad. & Tri. & AMM & Piéton & Cyc. & Auto & Total \\
\hline Trottoir & 62,0 & 47,4 & 34,6 & 48,3 & 50,9 & 38,1 & 78,1 & 51,4 \\
\hline Voie cyclable & 44,0 & 41,5 & 34,6 & 42,9 & 42,5 & 33,3 & 62,1 & 43,5 \\
\hline Chaussée & 38,3 & 32,3 & 30,8 & 35,0 & 34,7 & 21,8 & 40,9 & 32,5 \\
\hline Moyenne & 48,1 & 40,4 & 33,3 & 42,1 & 42,7 & 31,1 & 60,4 & 42,5 \\
\hline
\end{tabular}

Certains automobilistes ont ajouté que plusieurs AMM circulent au milieu de la chaussée et ne respectent pas les arrêts obligatoires et les feux de circulation. D'autres mentionnent qu'étant donné la différence de vitesse de déplacement, il est difficile d'avoir une cohabitation sur la route car c'est très difficile de les dépasser. D'autres ont mentionné les conflits d'espace lorsque les AMM circulent à deux de large. Beaucoup parlent des conflits de priorité, et certains mentionnent que les utilisateurs d'AMM ont parfois tendance à couper. Bref, beaucoup d'usagers ont des commentaires à formuler. Ils font surtout appel au vécu d'une personne et ne peuvent pas être généralisés.

Les utilisateurs d'AMM, les piétons, les cyclistes et les automobilistes ont été invités à se prononcer sur la nécessité de spécifier une vitesse autorisée pour les AMM, en fonction des trois principales infrastructures (Tableau 21). Peu de répondants voient l'utilité de spécifier une vitesse autorisée sur la chaussée $(33 \%)$. Cette nécessité se fait un peu plus sentir sur les voies cyclables, et davantage sur le trottoir, où la proportion atteint $51 \%$.

La ventilation par catégories démontre un très net rapprochement entre l'opinion des piétons et des utilisateurs d'AMM. Les automobilistes et les cyclistes ont une opinion différente du reste. Si l'on se fie à la moyenne, les premiers prônent beaucoup plus souvent le recours à une vitesse maximale, alors que les cyclistes sont moins enclins à ce genre de mesure. Par exemple, les automobilistes sont $78 \%$ à souhaiter que le législateur prescrive une vitesse maximale

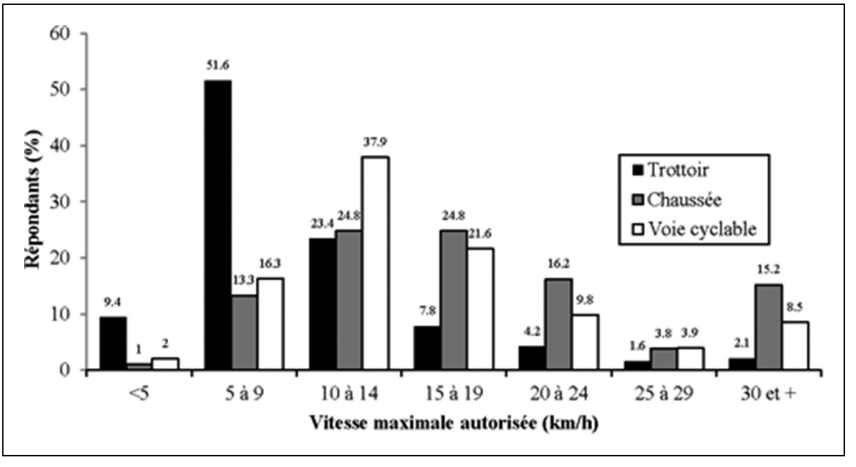

Fig. 10 Suggestions de vitesses maximales autorisées aux AMM

sur le trottoir, alors que les piétons, pourtant les premiers concernés, sont moins nombreux à le souhaiter (51\%).

Les répondants qui jugeaient nécessaire d'imposer une vitesse maximale devaient aussi spécifier une valeur absolue en kilomètres par heure. Pour simplifier le propos, la figure 10 présente des valeurs agrégées pour les quatre catégories de répondant. Sur le trottoir, la majorité des répondants (61\%) juge qu'un maximum de $9 \mathrm{~km} / \mathrm{h}$ est acceptable. Sur les voies cyclables ou sur la chaussée, le maximum qui devrait être autorisé est plus élevé.

En groupant l'ensemble des valeurs, une moyenne des vitesses a été calculée pour chaque catégorie de répondant, sur chaque type d'infrastructure (Tableau 22). On peut ainsi voir une gradation de la moyenne des vitesses 


\begin{tabular}{|c|c|c|c|c|c|}
\hline Infrastructure & AMM & Piéton & Cycliste & Auto & Total \\
\hline Trottoir & 7,7 & 10,7 & 11,2 & 7,9 & 8,8 \\
\hline Voie cyclable & 12,3 & 13,3 & 13,7 & 16,8 & 13,9 \\
\hline Chaussée & 14,4 & 15,6 & 15,8 & 25,6 & 17,1 \\
\hline
\end{tabular}

maximales suggérées, du trottoir $(9 \mathrm{~km} / \mathrm{h})$ vers la voie cyclable $(14 \mathrm{~km} / \mathrm{h})$ et la chaussée $(17 \mathrm{~km} / \mathrm{h})$. Le seul écart vraiment important concerne les automobilistes. Ceux-ci chiffrent leur moyenne à $26 \mathrm{~km} / \mathrm{h}$ sur la chaussée, soit $10 \mathrm{~km} / \mathrm{h}$ de plus que les trois autres catégories de répondant. Et aussi surprenant que cela puisse paraître, par rapport aux utilisateurs d'AMM, les piétons seraient en faveur de vitesses plus élevées sur les trottoirs pour ce genre d'appareil. Les piétons toléreraient en moyenne une vitesse de $11 \mathrm{~km} / \mathrm{h}$, tandis que les AMM se limiteraient eux-mêmes à $8 \mathrm{~km} / \mathrm{h}$. Il faut ici se rappeler qu'il s'agit d'opinions rapportées et on peut se demander quel est le degré de précision dans l'appréciation des vitesses maximales faite par les différentes clientèles.

\section{Discussion}

Au niveau de la capacité à marcher des utilisateurs d'AMM, deux constats s'imposent. Ceux qui se disent aptes à marcher sur une distance supérieure à un kilomètre ne représentent que $6 \%$ des utilisateurs d'AMM. Cette proportion contredit l'idée fréquemment lancée, à l'effet qu'une part importante d'utilisateurs d'AMM est tout à fait capable de marcher. Pour certains, le fait qu'une personne se lève de son appareil pour marcher peut laisser croire qu'elle n'a pas d'incapacité. Cette perception du public témoigne de la mauvaise compréhension de la réalité vécue par les utilisateurs d'AMM. En effet, $75 \%$ ne marchent pas ou peuvent marcher sur moins de 100 mètres, ce qui est un sérieux handicap à la mobilité piétonnière. De plus, $20 \%$ des utilisateurs de fauteuil roulant motorisé sont capables de marcher « un peu », ce qui suggère que l'usage d'une AMM est lié à un besoin profond, celui de rester mobile malgré la perte d'autonomie.

Par ailleurs, que la circulation sur le trottoir soit jugée non sécuritaire par un tiers de l'ensemble des répondants porte à réfléchir. En concordance avec le statut de piéton attribué partout dans le monde aux utilisateurs d'AMM, on pourrait s'attendre à ce que le trottoir soit jugé sécuritaire pour ces derniers et les autres usagers. Les infrastructures piétonnières pourraient, à tout le moins, être perçues comme étant tout aussi sécuritaires que les voies cyclables, sur lesquelles il est plus complexe de circuler en raison de la mixité et des vitesses pratiquées. Pour expliquer ce constat d'insécurité perçue sur les trottoirs, soulevé par $38 \%$ des utilisateurs d'AMM, ceux-ci énumèrent les difficultés de nature conceptuelle (largeur, dénivelé, dévers et joints esthétiques) et leur état de dégradation. Pour les autres clientèles, cette réalité est méconnue, et l'appréciation du risque, moindre même chez les piétons, vient davantage des craintes de conflits et du fait que les AMM impliquent un engin lourd et potentiellement rapide. Ces constats suggèrent-ils que le statut de piéton, accordé a priori et par défaut aux AMM, est en partie inadapté à leur condition, surtout lorsqu'on considère les caractéristiques physiques des trottoirs?

Même si l'AMM est par définition un moyen technique de pallier une incapacité à la marche, il semble que ce concept se heurte à des difficultés concrètes d'application. Ainsi, considérer l'utilisateur d'une AMM comme un piéton relève plus d'une position idéologique que pratique. En effet, de nombreux facteurs obligent à distinguer ces deux types d'usagers. Par exemple, les irritants et les inconforts associés à la circulation des AMM sur le trottoir ont été soulevés dans plusieurs études, et la présente vient les confirmer. Il importe cependant de rappeler que le trottoir québécois est souvent différent du trottoir européen, et est généralement constitué d'une succession de carrés de béton, avec autant de joints causant ruptures et vibrations pour les personnes en fauteuil, alors qu'en Europe, il est souvent asphalté donc sans discontinuité lorsqu'en bon état. Le confort offert par un aménagement cyclable et un trottoir n'est alors pas comparable si on comprend que tous ces joints offrent un confort moindre pour la circulation d'un engin ayant des roues. Un autre facteur qui distingue l'AMM du piéton est leur instabilité, notamment sur les trottoirs, et surtout dans le cas des triporteurs, qui ont tendance à verser facilement. Enfin, l'impossibilité de se déplacer latéralement constitue une autre contrainte aux AMM en comparaison avec les piétons.

Si l'utilisation du trottoir semble souvent problématique, la voie cyclable apparaît, quant à elle, comme l'infrastructure idéale. Toutefois, que fait l'usager lorsqu'il n'y a pas de voie cyclable ? Les usagers peu enclins à utiliser le trottoir n'ont alors qu'une seule alternative : prendre 
la chaussée. Les résultats montrent que cette option est privilégiée par plus de la moitié des utilisateurs d'AMM. Par contre, eux-mêmes et les autres clientèles s'accordent à dire que cette infrastructure est la moins sécuritaire de toutes ( $83 \%$ des répondants). Nous sommes donc en face d'une situation où bien que connaissant le danger inhérent à une pratique, les usagers l'adoptent quand même. D'autres motifs que la sécurité sont invoqués, tel le confort et le côté pratique, ce qu'ont mis en évidence les forums de discussion organisés au Québec sur cette question [14]. D'autre part, même si la chaussée est jugée comme peu sécuritaire, il n'y a pas encore de problème de sécurité démontré sur nos routes si on en juge par le peu d'utilisateurs d'AMM blessés gravement ou décédés suite à un accident survenu sur la chaussée.

Il serait intéressant de disposer de données permettant de comparer objectivement la sécurité des trottoirs et des chaussées. Or, cette comparaison est difficile à réaliser, car les accidents avec des véhicules routiers sont rapportés de façon systématique, alors que les blessures liées à l'utilisation du trottoir ne le sont pas. Répertorier ces dernières d'une manière systématique est pratiquement impossible au Québec. Des études sur les circonstances de survenue des blessures chez les usagers d'AMM, réalisées en Australie [17] et aux États-Unis [18], permettent d'estimer que la plupart des blessures sont le résultat de chutes, de $60 \%$ à $80 \%$. Au Québec, l'examen des données hospitalières n'a pas été réalisé, et les rapports de coroner montrent que sur 19 décès enregistrés en 15 ans, trois étaient liés à une chute de l'usager.

$\mathrm{Au}$ niveau du sens de la circulation, les résultats suggèrent que les utilisateurs d'AMM aillent dans le même sens que les cyclistes et les véhicules routiers. Ce concept risque cependant de se heurter à d'éventuelles difficultés d'application. Longtemps sensibilisés au fait qu'ils doivent circuler comme un piéton, donc se diriger dans le sens contraire s'ils sont sur la chaussée, les utilisateurs d'AMM ont pris l'habitude de circuler de cette façon. Leur demander du jour au lendemain de faire le contraire ne conviendrait qu'à $57 \%$ d'entre eux. Par contre, les piétons ( $85 \%)$ et les automobilistes (80\%) souhaitent que les AMM aillent dans le même sens que les véhicules. Quant à l'opinion des cyclistes ( $42 \%$ en faveur), elle s'explique peut-être par le fait qu'ils sont eux-mêmes nombreux à circuler dans le sens contraire aux véhicules, et ce possiblement pour diverses raisons : meilleur confort dû à une co-visibilité, continuité des cheminements. Cette réalité se reflète en Europe, notamment en France avec les contresens cyclables et en Belgique avec les sens uniques limités. De plus, la réglementation française permet de déroger à l'obligation de circuler du bord droit de la chaussée, notamment si la sécurité est mieux assurée de l'autre bord.
Ce questionnaire dresse aussi l'enjeu de la vitesse. Une expérience réalisée en laboratoire aux États-Unis montre que la vitesse pratiquée sur un fauteuil roulant motorisé n'aurait pas d'effet significatif sur le risque de chuter ou de perdre le contrôle [19]. Toutefois, le contexte expérimental diffère du contexte routier et deux notions méritent d'être distinguées : celle de la vitesse maximale théorique de l'appareil, et celle de la vitesse pratiquée. À l'exemple du Royaume-Uni, la limitation de la vitesse à la conception est une approche possible. D'autres choisissent plutôt d'établir un maximum autorisé sur le trottoir, par exemple $5 \mathrm{~km} / \mathrm{h}$ en Suède et $10 \mathrm{~km} / \mathrm{h}$ en Australie. Certaines juridictions s'en remettent au bon jugement de l'utilisateur de l'AMM et lui imposent circuler à l'allure du pas, ou de façon à ne pas compromettre la sécurité des usagers du trottoir (ex. : France, Belgique, Norvège, Danemark). Dans ce cas, on comprend bien que les règles sont basées sur une responsabilisation de l'usager. Même si le Royaume-Uni limite techniquement la vitesse des appareils pour sécuriser le trottoir, aucune limite de vitesse autre que celle qui est affichée pour tous n'est imposée sur la chaussée.

Au Québec, la Régie de l'assurance-maladie du Québec (RAMQ) octroie les AMM aux personnes qui rencontrent des critères spécifiques. La RAMQ choisit des modèles limités à la conception à $10 \mathrm{~km} / \mathrm{h}$, ou bride à cette vitesse les appareils plus rapides. Cette mesure du $10 \mathrm{~km} / \mathrm{h}$ systématique a été adoptée, et fait encore l'objet de discussions. En théorie, un véhicule autorisé à aller sur la chaussée devrait pouvoir excéder cette vitesse, afin d'assurer une meilleure cohabitation avec les clientèles plus rapides (ex. : vélos et véhicules). En fait, $10 \mathrm{~km} / \mathrm{h}$ est une vitesse qui semble trop basse pour la route et les voies cyclables. Il faudrait s'interroger, par souci de sécurité et de circulation harmonieuse, si le $10 \mathrm{~km} / \mathrm{h}$ n'implique pas un différentiel de vitesse trop élevé avec les autres clientèles qui empruntent ces infrastructures. À l'inverse, compte tenu des risques pour tous, on peut se demander s'il n'y a pas lieu d'introduire une vitesse maximale à la conception des AMM, comme il en existe une pour les vélos à assistance électrique.

Un peu plus de la moitié des piétons questionnés souhaiteraient une certaine limite sur le trottoir. Or, ils la fixeraient plus haute que les utilisateurs d'AMM eux-mêmes. En effet, les piétons toléreraient en moyenne $10,7 \mathrm{~km} / \mathrm{h}$ sur le trottoir, tandis que les utilisateurs d'AMM mentionnent en moyenne une vitesse maximale acceptable de $7,7 \mathrm{~km} / \mathrm{h}$. Sur les voies cyclables et sur la chaussée, la vitesse des AMM n'est pas une préoccupation, et c'est surtout la vitesse des véhicules motorisés qui peut poser problème.

L'autre problématique majeure est celle des équipements dont les AMM devraient être munis en particulier les 
phares. Encore là, la comparaison avec les piétons ne tient pas. Un piéton n'est pas tenu de marcher la nuit avec un phare à l'avant et une lumière à l'arrière. Bien qu'on recommande aux piétons d'être voyants dans l'obscurité, nul n'est obligé de porter des vêtements réfléchissants. Comme les piétons, les utilisateurs d'AMM échappent à ce secteur de la législation, à moins qu'ils ne soient dans un pays qui reconnaît leur légitimité à emprunter la chaussée dans le même sens que les véhicules. Qui dit droit d'emprunter la chaussée en tout temps dit obligation de munir l'appareil de certains équipements.

\section{Conclusion}

Cette enquête réalisée auprès des principales clientèles du réseau routier québécois met en lumière la perception face à d'éventuelles mesures légales impliquant les AMM et caractérise de façon globale l'usage actuel de ces appareils. Les résultats montrent que de nombreux aspects méritent d'être explorés encore plus à fond, tel le type d'infrastructure autorisé aux AMM, le sens de la circulation et la question de la vitesse. Bien que le modèle législatif adopté en Scandinavie et dans la plupart des pays européens puisse inspirer le futur cadre légal québécois, le projet pilote mis de l'avant par le Gouvernement permettra de mieux mesure la pratique, et d'évaluer l'impact des règles sur la sécurité. À terme de ce projet, il devrait être possible de porter un jugement mieux éclairé sur le bien-fondé de certaines règles testées dans le contexte québécois.

\section{Références}

1. France (2010) Code de la route. Partie réglementaire. Livre IV : L'usage des voies. Titre $1^{\text {er }}$ : Dispositions générales. Chap. II : Conduite des véhicules et circulation des piétons.

2. Belgique (2007) Arrêté royal relatif aux engins de déplacement. Moniteur Belge. Service public fédéral mobilité et transports 13:8830-8834.

3. Confédération suisse (2008) Ordonnance sur les règles de la circulation routière $(O C R)$ du 13 novembre 1962. 741.11, Mise à jour le $1^{\mathrm{er}}$ janvier 2008, $70 \mathrm{p}$.
4. Steyn PV, Chan AS (2008) Mobility Scooter Research Project Final Report. Centre for Education and Research on Aging, University College of the Fraser Valley, $114 \mathrm{p}$.

5. Barham P, Fereday D, Oxley P (2005) Review of Class 2 and Class 3 Powered Wheelchairs and Powered Scooters (Invalid Carriages). Transport \& Travel Research Ltd, PPAD 9/72/89, Department for Transport, United Kingdom, $62 \mathrm{p}$.

6. Su F, Bell MGH, Schmöcker J-D (2007) Mobility Scooter Usage In London-Results From the Scootability Project. 11e Conférence internationale sur la mobilité et le transport des personnes âgées ou à mobilité réduite, Montréal, 18-22 juin 2007.

7. Berndt A (2002) Scooters as a safe alternative for cars? Proceedings of the Road Safety Policing, Education and Enforcement Conference, Nov. 4-5, Adelaide, South Australia, p. 334-342.

8. Dolling M (2002) Motorised Scooters for Independent MobilityIssues of Equipment Design, Driving the Machine, and the Road Environment: A Challenge for the Safety of Impaired Users and the Community. Proceedings of the Road Safety Policing, Education and Enforcement Conference, Nov. 4-5, Adelaide, South Australia, p. 343-351.

9. Brighton C (2003) Rules of the road. Rehab Manag 16: 18-21.

10. Edwards K, McCluskey A (2010) A survey of adult power wheelchair and scooter users. Disabil Rehabil Assist Technol 5: 411-419.

11. Ulrich KT (2006) Estimating the technology frontier for personal electric vehicles. Transportation Research Part C: 448-462.

12. Gouvernement du Québec (2011) Code de la sécurité routière du Québec, Gazette Officielle du Québec, L.R.Q., c. C-24.2, À jour le $1^{\text {er }}$ février 2011.

13. Table québécoise de la sécurité routière (2007) Pour améliorer le bilan routier. Premier rapport de recommandations de la Table québécoise de la sécurité routière, $50 \mathrm{p}$.

14. Bruneau J-F, Maurice P, Lavoie M (2011) Avis de santé publique sur la circulation des aides à la mobilité motorisées sur le réseau routier. Institut national de santé publique du Québec, Québec, $143 \mathrm{p}$.

15. Bohannon RW (1997) Comfortable and maximum walking speed of adults aged 20-79 years: reference values and determinants. Age Ageing 26: 15-19.

16. Brandt A, Iwarsson S, Stahle A (2004) Older people's use of powered wheelchairs for activity and participation. $J$ Rehabil Med 36: 70-77.

17. Cassell E, Clapperton A (2006) Consumer product-related injury (2): Injury related to the use of motorised mobility scooters. Hazard 62: 1-16.

18. Xiang H, Chany AM, Smith GA (2006) Wheelchair related injuries treated in US emergency departments. Injury Prev 12: $8-11$.

19. Corfman TA, Cooper RA, Fitzgerald SG, Cooper R (2003) Tips and Falls During Electric-Powered Wheelchair Driving: Effects of Seatbelt Use, Legrests, and Driving Speed. Arch Phys Med Rehabil 84: 1797-1802. 